\title{
Supplement A: further proofs
}

Sigmoidal squasher is $\mathrm{N}$-admissible. Within the article we stated that the sigmoidal squasher $\sigma: \mathbb{R} \rightarrow[0,1], \sigma(x)=1 /(1+\exp (-x))$, satisfies Definition 3 for arbitrary $N \in \mathbb{N}$. This can be easily verified by checking the three properties therein. At first, $\sigma$ is obviously continuously differentiable and its derivative can be written as

$$
\begin{aligned}
\frac{\partial \sigma}{\partial x}(x) & =-(1+\exp (-x))^{-2} \cdot(-\exp (-x) \\
& =\frac{\exp (-x)}{1+\exp (-x)} \cdot \frac{1}{1+\exp (-x)} \\
& =\left(1-\frac{1}{1+\exp (-x)}\right) \cdot \frac{1}{1+\exp (-x)} \\
& =(1-\sigma(x)) \cdot \sigma(x) .
\end{aligned}
$$

Consequently, thanks to the product rule of differentiation, all derivatives of $\sigma$ are polynomials in $\sigma$. That implies property $(i)$ in Definition 3 because $\sigma$ is bounded by 0 and 1 . Furthermore, polynomials (which are not the null function) have only a finite number of roots within $(0,1)$ and $\sigma$ reaches all values in this interval. Thus, property $(i i)$ is satisfied as well. Finally, consider an arbitrary $x>0$. Then $x \leq \exp (x)+1$ holds. So we can conclude

$$
\begin{aligned}
& x \leq \exp (x)+1 \\
\Leftrightarrow & x \cdot \exp (-x) \leq 1+\exp (-x) \\
\Leftrightarrow & \frac{\exp (-x)}{1+\exp (-x)} \leq \frac{1}{x} \\
\Leftrightarrow & 1-\frac{1}{1+\exp (-x)} \leq \frac{1}{x} .
\end{aligned}
$$

The left-hand side is equivalent to $|\sigma(x)-1|$, which proves the first relation mentioned in property (iii). The second relation follows in the same way because $\sigma$ is symmetric to the point $\left(0 \mid \frac{1}{2}\right)$.

Proof of Lemma 1. In the proof we use the following error decomposition:

$$
\begin{aligned}
\int \mid m_{n}(x)- & \left.m(x)\right|^{2} \mu(d x) \\
= & {\left[\mathbf{E}\left\{\left|m_{n}(X)-Y\right|^{2} \mid \mathcal{D}_{n}\right\}-\mathbf{E}\left\{|m(X)-Y|^{2}\right\}\right.} \\
& \left.\quad-\left(\mathbf{E}\left\{\left|m_{n}(X)-T_{\beta_{n}} Y\right|^{2} \mid \mathcal{D}_{n}\right\}-\mathbf{E}\left\{\left|m_{\beta_{n}}(X)-T_{\beta_{n}} Y\right|^{2}\right\}\right)\right] \\
+ & {\left[\mathbf{E}\left\{\left|m_{n}(X)-T_{\beta_{n}} Y\right|^{2} \mid \mathcal{D}_{n}\right\}-\mathbf{E}\left\{\left|m_{\beta_{n}}(X)-T_{\beta_{n}} Y\right|^{2}\right\}\right.} \\
& \left.-2 \cdot \frac{1}{n} \sum_{i=1}^{n}\left(\left|m_{n}\left(X_{i}\right)-T_{\beta_{n}} Y_{i}\right|^{2}-\left|m_{\beta_{n}}\left(X_{i}\right)-T_{\beta_{n}} Y_{i}\right|^{2}\right)\right]
\end{aligned}
$$




$$
\begin{aligned}
& +\left[2 \cdot \frac{1}{n} \sum_{i=1}^{n}\left|m_{n}\left(X_{i}\right)-T_{\beta_{n}} Y_{i}\right|^{2}-2 \cdot \frac{1}{n} \sum_{i=1}^{n}\left|m_{\beta_{n}}\left(X_{i}\right)-T_{\beta_{n}} Y_{i}\right|^{2}\right. \\
& \left.\quad-\left(2 \cdot \frac{1}{n} \sum_{i=1}^{n}\left|m_{n}\left(X_{i}\right)-Y_{i}\right|^{2}-2 \cdot \frac{1}{n} \sum_{i=1}^{n}\left|m\left(X_{i}\right)-Y_{i}\right|^{2}\right)\right] \\
& +\left[2\left(\frac{1}{n} \sum_{i=1}^{n}\left|m_{n}\left(X_{i}\right)-Y_{i}\right|^{2}-\frac{1}{n} \sum_{i=1}^{n}\left|m\left(X_{i}\right)-Y_{i}\right|^{2}\right)\right] \\
& =\sum_{i=1}^{4} T_{i, n},
\end{aligned}
$$

where $T_{\beta_{n}} Y$ is the truncated version of $Y$ and $m_{\beta_{n}}$ is the regression function of $T_{\beta_{n}} Y$, i.e.,

$$
m_{\beta_{n}}(x)=\mathbf{E}\left\{T_{\beta_{n}} Y \mid X=x\right\} .
$$

We start with bounding $T_{1, n}$. By using $a^{2}-b^{2}=(a-b)(a+b)$ we get

$$
\begin{aligned}
T_{1, n}= & \mathbf{E}\left\{\left|m_{n}(X)-Y\right|^{2}-\left|m_{n}(X)-T_{\beta_{n}} Y\right|^{2} \mid \mathcal{D}_{n}\right\} \\
& -\mathbf{E}\left\{|m(X)-Y|^{2}-\left|m_{\beta_{n}}(X)-T_{\beta_{n}} Y\right|^{2}\right\} \\
= & \mathbf{E}\left\{\left(T_{\beta_{n}} Y-Y\right)\left(2 m_{n}(X)-Y-T_{\beta_{n}} Y\right) \mid \mathcal{D}_{n}\right\} \\
& -\mathbf{E}\left\{\left(\left(m(X)-m_{\beta_{n}}(X)\right)+\left(T_{\beta_{n}} Y-Y\right)\right)\left(m(X)+m_{\beta_{n}}(X)-Y-T_{\beta_{n}} Y\right)\right\} \\
= & T_{5, n}+T_{6, n} .
\end{aligned}
$$

With the Cauchy-Schwarz inequality and

$$
I_{\left\{|Y|>\beta_{n}\right\}} \leq \frac{\exp \left(c_{1} / 2 \cdot|Y|^{2}\right)}{\exp \left(c_{1} / 2 \cdot \beta_{n}^{2}\right)}
$$

we conclude

$$
\begin{aligned}
\left|T_{5, n}\right| \leq & \sqrt{\mathbf{E}\left\{\left|T_{\beta_{n}} Y-Y\right|^{2}\right\}} \cdot \sqrt{\mathbf{E}\left\{\left|2 m_{n}(X)-Y-T_{\beta_{n}} Y\right|^{2} \mid \mathcal{D}_{n}\right\}} \\
\leq & \sqrt{\mathbf{E}\left\{|Y|^{2} \cdot I_{\left\{|Y|>\beta_{n}\right\}}\right\}} \cdot \sqrt{\mathbf{E}\left\{2 \cdot\left|2 m_{n}(X)-T_{\beta_{n}} Y\right|^{2}+2 \cdot|Y|^{2} \mid \mathcal{D}_{n}\right\}} \\
\leq & \sqrt{\mathbf{E}\left\{|Y|^{2} \cdot \frac{\exp \left(c_{1} / 2 \cdot|Y|^{2}\right)}{\exp \left(c_{1} / 2 \cdot \beta_{n}^{2}\right)}\right\}} \\
& \cdot \sqrt{\mathbf{E}\left\{2 \cdot\left|2 m_{n}(X)-T_{\beta_{n}} Y\right|^{2} \mid \mathcal{D}_{n}\right\}+2 \mathbf{E}\left\{|Y|^{2}\right\}} \\
\leq & \sqrt{\mathbf{E}\left\{|Y|^{2} \cdot \exp \left(c_{1} / 2 \cdot|Y|^{2}\right)\right\}} \cdot \exp \left(-\frac{c_{1} \cdot \beta_{n}^{2}}{4}\right) \cdot \sqrt{2\left(3 \beta_{n}\right)^{2}+2 \mathbf{E}\left\{|Y|^{2}\right\}} .
\end{aligned}
$$

With $x \leq \exp (x)$ for $x \in \mathbb{R}$ we get

$$
|Y|^{2} \leq \frac{2}{c_{1}} \cdot \exp \left(\frac{c_{1}}{2} \cdot|Y|^{2}\right)
$$


and hence $\sqrt{\mathbf{E}\left\{|Y|^{2} \cdot \exp \left(c_{1} / 2 \cdot|Y|^{2}\right)\right\}}$ is bounded by

$$
\mathbf{E}\left(\frac{2}{c_{1}} \cdot \exp \left(c_{1} / 2 \cdot|Y|^{2}\right) \cdot \exp \left(c_{1} / 2 \cdot|Y|^{2}\right)\right) \leq \mathbf{E}\left(\frac{2}{c_{1}} \cdot \exp \left(c_{1} \cdot|Y|^{2}\right)\right) \leq c_{38}
$$

which is less than infinity by the assumptions of the lemma. Furthermore the third term is bounded by $\sqrt{18 \beta_{n}^{2}+c_{39}}$ because

$$
\mathbf{E}\left(|Y|^{2}\right) \leq \mathbf{E}\left(1 / c_{1} \cdot \exp \left(c_{1} \cdot|Y|^{2}\right) \leq c_{39}<\infty,\right.
$$

which follows again as above. With the setting $\beta_{n}=c_{26} \cdot \log (n)$ it follows for some constants $c_{40}, c_{41}>0$ that

$$
\left|T_{5, n}\right| \leq \sqrt{c_{38}} \cdot \exp \left(-c_{40} \cdot \log (n)^{2}\right) \cdot \sqrt{\left(18 \cdot c_{26} \cdot \log (n)^{2}+c_{39}\right)} \leq c_{41} \cdot \frac{\log (n)}{n} .
$$

From the Cauchy-Schwarz inequality we get

$$
\begin{gathered}
T_{6, n} \leq \sqrt{2 \cdot \mathbf{E}\left\{\left|\left(m(X)-m_{\beta_{n}}(X)\right)\right|^{2}\right\}+2 \cdot \mathbf{E}\left\{\left|\left(T_{\beta_{n}} Y-Y\right)\right|^{2}\right\}} \\
\cdot \sqrt{\mathbf{E}\left\{\left|m(X)+m_{\beta_{n}}(X)-Y-T_{\beta_{n}} Y\right|^{2}\right\}},
\end{gathered}
$$

where we can bound the second factor on the right-hand side in the above inequality in the same way we have bounded the second factor from $T_{5, n}$, because by assumption $\|m\|_{\infty}$ is bounded and furthermore $m_{\beta_{n}}$ is bounded by $\beta_{n}$. Thus we get for some constant $c_{42}>0$

$$
\sqrt{\mathbf{E}\left\{\left|m(X)+m_{\beta_{n}}(X)-Y-T_{\beta_{n}} Y\right|^{2}\right\}} \leq c_{42} \cdot \log (n) .
$$

Next we consider the first term. With Jensen's inequality it follows that

$$
\mathbf{E}\left\{\left|m(X)-m_{\beta_{n}}(X)\right|^{2}\right\} \leq \mathbf{E}\left\{\mathbf{E}\left(\left|Y-T_{\beta_{n}} Y\right|^{2} \mid X\right)\right\}=\mathbf{E}\left\{\left|Y-T_{\beta_{n}} Y\right|^{2}\right\} .
$$

Hence we get

$$
T_{6, n} \leq \sqrt{4 \cdot \mathbf{E}\left\{\left|Y-T_{\beta_{n}} Y\right|^{2}\right\}} \cdot c_{42} \cdot \log (n)
$$

and therefore with the calculations from $T_{5, n}$ it follows that $T_{6, n} \leq c_{43} \cdot \log (n) / n$ for some constant $c_{43}>0$. Altogether we get

$$
T_{1, n} \leq c_{44} \cdot \frac{\log (n)}{n}
$$


for some constant $c_{44}>0$.

Next we consider $T_{2, n}$ and conclude for $t>0$

$$
\begin{aligned}
\mathbf{P}\left\{T_{2, n}>t\right\} \leq \mathbf{P} & \left\{\exists f \in T_{\beta_{n}, \operatorname{supp}(X)} \mathcal{F}_{n}: \mathbf{E}\left(\left|\frac{f(X)}{\beta_{n}}-\frac{T_{\beta_{n}} Y}{\beta_{n}}\right|^{2}\right)-\mathbf{E}\left(\left|\frac{m_{\beta_{n}}(X)}{\beta_{n}}-\frac{T_{\beta_{n}} Y}{\beta_{n}}\right|^{2}\right)\right. \\
& -\frac{1}{n} \sum_{i=1}^{n}\left(\left|\frac{f\left(X_{i}\right)}{\beta_{n}}-\frac{T_{\beta_{n}} Y_{i}}{\beta_{n}}\right|^{2}-\left|\frac{m_{\beta_{n}}\left(X_{i}\right)}{\beta_{n}}-\frac{T_{\beta_{n}} Y_{i}}{\beta_{n}}\right|^{2}\right) \\
& \left.>\frac{1}{2}\left(\frac{t}{\beta_{n}^{2}}+\mathbf{E}\left(\left|\frac{f(X)}{\beta_{n}}-\frac{T_{\beta_{n}} Y}{\beta_{n}}\right|^{2}\right)-\mathbf{E}\left(\left|\frac{m_{\beta_{n}}(X)}{\beta_{n}}-\frac{T_{\beta_{n}} Y}{\beta_{n}}\right|^{2}\right)\right)\right\},
\end{aligned}
$$

where $T_{\beta_{n}, \operatorname{supp}(X)} \mathcal{F}_{n}$ is defined as $\left\{T_{\beta_{n}} f \cdot 1_{\operatorname{supp}(X)}: f \in \mathcal{F}_{n}\right\}$. Theorem 11.4 in Györfi et al. (2002) and the relation $\mathcal{N}\left(\delta,\left\{\frac{1}{\beta_{n}} g: g \in \mathcal{G}\right\},\|\cdot\|_{\infty, \operatorname{supp}(X)}\right) \leq \mathcal{N}\left(\delta \cdot \beta_{n}, \mathcal{G},\|\cdot\|_{\infty, \operatorname{supp}(X)}\right)$ for an arbitrary function space $\mathcal{G}$ and $\delta>0$ lead to

$$
\mathbf{P}\left\{T_{2, n}>t\right\} \leq 14 \cdot \mathcal{N}\left(\frac{t}{80 \cdot \beta_{n}}, \mathcal{F}_{n},\|\cdot\|_{\infty, \operatorname{supp}(X)}\right) \cdot \exp \left(-\frac{n}{5136 \cdot \beta_{n}^{2}} \cdot t\right) .
$$

Since the covering number and the exponential factor are decreasing in $t$, we can conclude for $\varepsilon_{n} \geq \frac{80}{n}$

$$
\begin{aligned}
\mathbf{E}\left(T_{2, n}\right) & \leq \varepsilon_{n}+\int_{\varepsilon_{n}}^{\infty} \mathbf{P}\left\{T_{2, n}>t\right\} d t \\
& \leq \varepsilon_{n}+14 \cdot \mathcal{N}\left(\frac{1}{n \cdot \beta_{n}}, \mathcal{F}_{n},\|\cdot\|_{\infty, \operatorname{supp}(X)}\right) \cdot \exp \left(-\frac{n}{5136 \cdot \beta_{n}^{2}} \cdot \varepsilon_{n}\right) \cdot \frac{5136 \cdot \beta_{n}^{2}}{n} .
\end{aligned}
$$

Choosing

$$
\varepsilon_{n}=\frac{5136 \cdot \beta_{n}^{2}}{n} \cdot \log \left(14 \cdot \mathcal{N}\left(\frac{1}{n \cdot \beta_{n}}, \mathcal{F}_{n},\|\cdot\|_{\infty, \operatorname{supp}(X)}\right)\right)
$$

(which satisfies the necessary condition $\varepsilon_{n} \geq \frac{80}{n}$ if the constant $c_{5}$ in the definition of $\beta_{n}$ is not too small) minimizes the right-hand side and implies

$$
\mathbf{E}\left(T_{2, n}\right) \leq \frac{c_{7} \cdot \log (n)^{2} \cdot \log \left(\mathcal{N}\left(\frac{1}{n \cdot \beta_{n}}, \mathcal{F}_{n},\|\cdot\|_{\infty, \operatorname{supp}(X)}\right)\right)}{n} .
$$

By bounding $T_{3, n}$ similarly to $T_{1, n}$ we get

$$
\mathbf{E}\left(T_{3, n}\right) \leq c_{45} \cdot \frac{\log (n)}{n}
$$

for some large enough constant $c_{45}>0$ and hence we get in total

$$
\mathbf{E}\left(\sum_{i=1}^{3} T_{i, n}\right) \leq \frac{c_{46} \cdot \log (n)^{2} \cdot \log \left(\mathcal{N}\left(\frac{1}{n \cdot \beta_{n}}, \mathcal{F}_{n},\|\cdot\|_{\infty, \operatorname{supp}(X)}\right)\right)}{n}
$$


for some sufficient large constant $c_{46}>0$.

We finish the proof by bounding $T_{4, n}$. Let $A_{n}$ be the event, that there exists $i \in$ $\{1, \ldots, n\}$ such that $\left|Y_{i}\right|>\beta_{n}$ and let $I_{A_{n}}$ be the indicator function of $A_{n}$. Then we get

$$
\begin{aligned}
\mathbf{E}\left(T_{4, n}\right) \leq & 2 \cdot \mathbf{E}\left(\frac{1}{n} \sum_{i=1}^{n}\left|m_{n}\left(X_{i}\right)-Y_{i}\right|^{2} \cdot I_{A_{n}}\right) \\
& +2 \cdot \mathbf{E}\left(\frac{1}{n} \sum_{i=1}^{n}\left|m_{n}\left(X_{i}\right)-Y_{i}\right|^{2} \cdot I_{A_{n}^{c}}-\frac{1}{n} \sum_{i=1}^{n}\left|m\left(X_{i}\right)-Y_{i}\right|^{2}\right) \\
= & 2 \cdot \mathbf{E}\left(\left|m_{n}\left(X_{1}\right)-Y_{1}\right|^{2} \cdot I_{A_{n}}\right) \\
& +2 \cdot \mathbf{E}\left(\frac{1}{n} \sum_{i=1}^{n}\left|m_{n}\left(X_{i}\right)-Y_{i}\right|^{2} \cdot I_{A_{n}^{c}}-\frac{1}{n} \sum_{i=1}^{n}\left|m\left(X_{i}\right)-Y_{i}\right|^{2}\right) \\
= & T_{7, n}+T_{8, n} .
\end{aligned}
$$

With the Cauchy-Schwarz inequality we get for $T_{7, n}$

$$
\begin{aligned}
\frac{1}{2} \cdot T_{7, n} & \leq \sqrt{\mathbf{E}\left(\left(\left|m_{n}\left(X_{1}\right)-Y_{1}\right|^{2}\right)^{2}\right)} \cdot \sqrt{\mathbf{P}\left(A_{n}\right)} \\
& \leq \sqrt{\mathbf{E}\left(\left(2\left|m_{n}\left(X_{1}\right)\right|^{2}+2\left|Y_{1}\right|^{2}\right)^{2}\right)} \cdot \sqrt{n \cdot \mathbf{P}\left\{\left|Y_{1}\right|>\beta_{n}\right\}} \\
& \leq \sqrt{\mathbf{E}\left(8\left|m_{n}\left(X_{1}\right)\right|^{4}+8\left|Y_{1}\right|^{4}\right)} \cdot \sqrt{n \cdot \frac{\mathbf{E}\left(\exp \left(c_{1} \cdot\left|Y_{1}\right|^{2}\right)\right)}{\exp \left(c_{1} \cdot \beta_{n}^{2}\right)}},
\end{aligned}
$$

where the last inequality follows from inequality (17). With $x \leq \exp (x)$ for $x \in \mathbb{R}$ we get

$$
\begin{aligned}
\mathbf{E}\left(|Y|^{4}\right) & =\mathbf{E}\left(|Y|^{2} \cdot|Y|^{2}\right) \leq \mathbf{E}\left(\frac{2}{c_{1}} \cdot \exp \left(\frac{c_{1}}{2} \cdot|Y|^{2}\right) \cdot \frac{2}{c_{1}} \cdot \exp \left(\frac{c_{1}}{2} \cdot|Y|^{2}\right)\right) \\
& =\frac{4}{c_{1}^{2}} \cdot \mathbf{E}\left(\exp \left(c_{1} \cdot|Y|^{2}\right)\right),
\end{aligned}
$$

which is less than infinity by condition (12) of the lemma. Furthermore $\left\|m_{n}\right\|_{\infty}$ is bounded by $\beta_{n}$ and therefore the first factor is bounded by

$$
c_{47} \cdot \beta_{n}^{2}=c_{48} \cdot \log (n)^{2}
$$

for some constant $c_{48}>0$. The second factor is bounded by $1 / n$, because by the assumptions of the lemma $\mathbf{E}\left(\exp \left(c_{1} \cdot\left|Y_{1}\right|^{2}\right)\right)$ is bounded by some constant $c_{49}<\infty$ and hence we get

$$
\sqrt{n \cdot \frac{\mathbf{E}\left(\exp \left(c_{1} \cdot\left|Y_{1}\right|^{2}\right)\right)}{\exp \left(c_{1} \cdot \beta_{n}^{2}\right)}} \leq \sqrt{n} \cdot \frac{\sqrt{c_{49}}}{\sqrt{\exp \left(c_{1} \cdot \beta_{n}^{2}\right)}} \leq \frac{\sqrt{n} \cdot \sqrt{c_{49}}}{\exp \left(\left(c_{1} \cdot c_{26}^{2} \cdot \log (n)^{2}\right) / 2\right)} .
$$

Since $\exp \left(-c \cdot \log (n)^{2}\right)=O\left(n^{-2}\right)$ for any $c>0$, we get altogether

$$
T_{7, n} \leq c_{50} \cdot \frac{\log (n)^{2} \sqrt{n}}{n^{2}} \leq c_{51} \cdot \frac{\log (n)^{2}}{n} .
$$


With the definition of $A_{n}^{c}$ and $\tilde{m}_{n}$ defined as in the assumptions of this lemma we conclude

$$
\begin{aligned}
T_{8, n} & \leq 2 \cdot \mathbf{E}\left(\frac{1}{n} \sum_{i=1}^{n}\left|\tilde{m}_{n}\left(X_{i}\right)-Y_{i}\right|^{2} \cdot I_{A_{n}^{c}}-\frac{1}{n} \sum_{i=1}^{n}\left|m\left(X_{i}\right)-Y_{i}\right|^{2}\right) \\
& \leq 2 \cdot \mathbf{E}\left(\frac{1}{n} \sum_{i=1}^{n}\left|\tilde{m}_{n}\left(X_{i}\right)-Y_{i}\right|^{2}-\frac{1}{n} \sum_{i=1}^{n}\left|m\left(X_{i}\right)-Y_{i}\right|^{2}\right) \\
& \leq 2 \cdot \mathbf{E}\left(\inf _{f \in \mathcal{F}_{n}} \frac{1}{n} \sum_{i=1}^{n}\left|f\left(X_{i}\right)-Y_{i}\right|^{2}-\frac{1}{n} \sum_{i=1}^{n}\left|m\left(X_{i}\right)-Y_{i}\right|^{2}\right),
\end{aligned}
$$

because $\left|T_{\beta} z-y\right| \leq|z-y|$ holds for $|y| \leq \beta$. Hence

$$
\mathbf{E}\left(T_{4, n}\right) \leq c_{51} \cdot \frac{\log (n)^{2}}{n}+2 \cdot \mathbf{E}\left(\inf _{f \in \mathcal{F}_{n}} \frac{1}{n} \sum_{i=1}^{n}\left|f\left(X_{i}\right)-Y_{i}\right|^{2}-\frac{1}{n} \sum_{i=1}^{n}\left|m\left(X_{i}\right)-Y_{i}\right|^{2}\right)
$$

holds. If we choose an $f^{*} \in \mathcal{F}_{n}$ which satisfies

$$
\int\left|f^{*}(x)-m(x)\right|^{2} \mathbf{P}_{X}(d x) \leq \inf _{f \in \mathcal{F}_{n}} \int|f(x)-m(x)|^{2} \mathbf{P}_{X}(d x)+\frac{1}{n},
$$

we can conclude

$$
\begin{aligned}
& \mathbf{E}\left(\inf _{f \in \mathcal{F}_{n}} \frac{1}{n} \sum_{i=1}^{n}\left|f\left(X_{i}\right)-Y_{i}\right|^{2}-\frac{1}{n} \sum_{i=1}^{n}\left|m\left(X_{i}\right)-Y_{i}\right|^{2}\right) \\
& \leq \mathbf{E}\left\{\frac{1}{n} \sum_{i=1}^{n}\left|f^{*}\left(X_{i}\right)-Y_{i}\right|^{2}-\frac{1}{n} \sum_{i=1}^{n}\left|m\left(X_{i}\right)-Y_{i}\right|^{2}\right\} \\
& =\mathbf{E}\left\{\left|f^{*}(X)-Y\right|^{2}\right\}-\mathbf{E}\left\{|m(X)-Y|^{2}\right\} \\
& =\mathbf{E}\left\{\left|f^{*}(X)-m(X)\right|^{2}\right\}+\mathbf{E}\left\{|m(X)-Y|^{2}\right\}-\mathbf{E}\left\{|m(X)-Y|^{2}\right\} \\
& \leq \inf _{f \in \mathcal{F}_{n}} \int|f(x)-m(x)|^{2} \mathbf{P}_{X}(d x)+\frac{1}{n} .
\end{aligned}
$$

In combination with the other considerations in the proof this implies the assertion of Lemma 1.

In order to prove Lemma 2, we will apply the following lemma.

Lemma 9. Let $l \in \mathbb{N}_{0}$ and let $\sigma_{r}: \mathbb{R} \rightarrow \mathbb{R}$ for $r=1, \ldots, l+1$ be Lipschitz continuous functions with Lipschitz constant $L \geq 1$, which satisfy

$$
\left|\sigma_{r}(x)\right| \leq L \cdot \max \{|x|, 1\} \quad(x \in \mathbb{R}) .
$$

Let $K_{0}=d, K_{r} \in \mathbb{N}$ for $r \in\{1, \ldots, l\}$ and $K_{l+1}=1$. For $r \in\{1, \ldots, l+1\}$ and $i \in\left\{1, \ldots, K_{r}\right\}$ define recursively

$$
f_{i}^{(r)}(x)=\sigma_{r}\left(\sum_{j=1}^{K_{r-1}} c_{i, j}^{(r-1)} \cdot f_{j}^{(r-1)}(x)+c_{i, 0}^{(r-1)}\right)
$$


and

$$
\bar{f}_{i}^{(r)}(x)=\sigma_{r}\left(\sum_{j=1}^{K_{r-1}} \bar{c}_{i, j}^{(r-1)} \cdot \bar{f}_{j}^{(r-1)}(x)+\bar{c}_{i, 0}^{(r-1)}\right),
$$

where $c_{i, 0}^{(r-1)}, \bar{c}_{i, 0}^{(r-1)}, \ldots, c_{i, K_{r-1}}^{(r-1)}, \bar{c}_{i, K_{r-1}}^{(r-1)} \in \mathbb{R}$, and $f_{j}^{(0)}(x)=\bar{f}_{j}^{(0)}(x)=x^{(j)}$. Furthermore, set

$$
\bar{C}=\max _{\substack{r=0, \ldots, l, i=1, \ldots, K_{r+1}, j=1, \ldots, K_{r}}} \max \left\{\left|c_{i, j}^{(r)}\right|,\left|\bar{c}_{i, j}^{(r)}\right|, 1\right\} .
$$

Then

$$
\begin{aligned}
& \left|f_{1}^{(l+1)}(x)-\bar{f}_{1}^{(l+1)}(x)\right| \\
& \leq(l+1) \cdot L^{l+1} \cdot \prod_{r=0}^{l}\left(K_{r}+1\right) \cdot \bar{C}^{l} \cdot \max \left\{\|x\|_{\infty}, 1\right\} \cdot \max _{\substack{r=0, \ldots, l, i=1, \ldots, K_{r+1}, j=0, \ldots, K_{r}}}\left|c_{i, j}^{(r)}-\bar{c}_{i, j}^{(r)}\right|
\end{aligned}
$$

for any $x \in \mathbb{R}^{d}$.

Proof. See Lemma 5 in Bauer et al. (2017).

Proof of Lemma 2. At first, we notice the space $\mathcal{H}^{(l)}$ (with $l>0$ ) can be expressed as $\mathcal{H}^{(l)}=\left\{h: \mathbb{R}^{d} \rightarrow \mathbb{R}: h(x)=\sum_{k=1}^{K} \sigma_{i d}\left(g_{k}\left(\sigma_{i d}\left(f_{1, k}(x)\right), \ldots, \sigma_{i d}\left(f_{d^{*}, k}(x)\right)\right)\right) \quad\left(x \in \mathbb{R}^{d}\right)\right.$
for some $g_{k} \in \mathcal{F}_{M^{*}, d^{*}, d^{*}, \alpha, \beta, \gamma}^{(\text {neural networks })}$ and $\left.f_{j, k} \in \mathcal{H}^{(l-1)}\right\}$, where $\sigma_{i d}: \mathbb{R} \rightarrow \mathbb{R}$ is the identity $\sigma_{i d}(x)=x$ for all $x \in \mathbb{R}$. Furthermore, all $g \in$ $\mathcal{F}_{M^{*}, d^{*}, d^{*}, \alpha}^{(\text {neural networks })}$ can be written as

$$
\begin{aligned}
g(x) & =\sum_{i=1}^{M^{*}} \mu_{i} \cdot \sigma\left(\sum_{l=1}^{4 d^{*}} \lambda_{i, l} \cdot \sigma\left(\sum_{v=1}^{d^{*}} \theta_{i, l, v} \cdot x^{(v)}+\theta_{i, l, 0}\right)+\lambda_{i, 0}\right) \\
& =\sum_{i=1}^{M^{*}} \mu_{i} \cdot \sigma\left(\sum_{\substack{l=1, \ldots, 4 d^{*} \\
\bar{i}=1, \ldots, M^{*}}} \lambda_{i, \bar{i}, l} \cdot \sigma\left(\sum_{v=1}^{d^{*}} \theta_{\bar{i}, l, v} \cdot x^{(v)}+\theta_{\bar{i}, l, 0}\right)+\lambda_{i, \bar{i}, 0}\right)
\end{aligned}
$$

where the new coefficients are defined by

$$
\lambda_{i, \bar{i}, l}:= \begin{cases}\lambda_{i, l} & \text { if } \bar{i}=i \\ 0 & \text { otherwise }\end{cases}
$$

for all $i, \bar{i} \in\left\{1, \ldots, M^{*}\right\}$ and $l \in\left\{0, \ldots, 4 d^{*}\right\}$ (which works analogously for $h \in \mathcal{H}^{(0)}$ ). Respecting the above representations, all the functions $\sigma_{i d}(h)=h$ for $h \in \mathcal{H}^{(l)}$ comply 
with the structure of the functions $f_{1}^{(l+1)}$ in Lemma 9 , if we use the following specifications of the parameters in that lemma: The Lipschitz constant $L$ is chosen as the maximum of the Lipschitz constants of $\sigma_{i d}$ (which is obviously 1 ) and of the $N$-admissible sigmoidal function $\sigma$. Thus, property (19) is satisfied due to $\|\sigma\|_{\infty} \leq 1, L \geq 1$, and $\left|\sigma_{i d}(x)\right|=|x|$. The parameter $l$ in this lemma is $4 l+2$ (regarding the $l$ in $\mathcal{H}^{(l)}$ above) and the parameters $K_{r}$ with $r=0, \ldots, l$ take repeatedly the values $\tilde{d}, 4 \cdot d^{*} \cdot M^{*}, M^{*}$ and $K$ one after another, where $\tilde{d}$ is equal to $d^{*}$ except for $K_{0}$, where it is $d$. Since all the coefficients $c_{i, j}^{(r)}$ with $r=0, \ldots, l, i=1, \ldots, K_{r+1}, j=1, \ldots, K_{r}$ (using $K_{l+1}=1$ again) are 0,1 , or one of the $\mu_{i}, \lambda_{i, l}, \theta_{i, l, v}$ in the definition of $\mathcal{F}_{M^{*}, d^{*}, d, \alpha}^{(\text {neetworks })}$, we can use $\bar{C}=\max \{\alpha, 1\}$ for $n$ sufficiently large.

Let $h$ and $\bar{h}$ be functions in $\mathcal{H}^{(l)}$. Since they comply with the structure of the functions in Lemma 9 according to the above argumentation, we can conclude

$$
\begin{aligned}
& \|h-\bar{h}\|_{\infty,\left[-a_{n}, a_{n}\right]^{d}} \\
& \leq(4 l+3) \cdot L^{4 l+3} \cdot\left(4 \cdot d^{*} \cdot M^{*}+1\right)^{4 l+3} \cdot \max \{\alpha, 1\}^{4 l+2} \\
& \cdot \max \left\{a_{n}, 1\right\} \cdot \max _{\substack{r=0, \ldots, l, i=1, \ldots, K_{r+1} \\
j=0, \ldots, K_{r}}}\left|c_{i, j}^{(r)}-\bar{c}_{i, j}^{(r)}\right| \\
& \leq n^{c_{5} 2} \cdot \max _{\substack{r=0, \ldots, l, i=1, \ldots, K_{r+1}, j=0, \ldots, K_{r}}}\left|c_{i, j}^{(r)}-\bar{c}_{i, j}^{(r)}\right|
\end{aligned}
$$

for $n$ sufficiently large and an adequately chosen $c_{52}>0$ thanks to $\max \left\{a_{n}, \alpha, M^{*}\right\} \leq n^{c 9}$. Thus, if we consider an arbitrary $h \in \mathcal{H}^{(l)}$, it suffices to choose the coefficients $\bar{c}_{i, j}^{(r)}$ of a function $\bar{h} \in \mathcal{H}^{(l)}$ such that

$$
\left|c_{i, j}^{(r)}-\bar{c}_{i, j}^{(r)}\right| \leq \frac{\varepsilon_{n}}{n^{c_{52}}}
$$

holds for all possible indices, in order to satisfy $\|h-\bar{h}\|_{\infty,\left[-a_{n}, a_{n}\right]^{d}} \leq \varepsilon_{n}$. These coefficients $c_{i, j}^{(r)}$ have to take values in $[-\alpha, \alpha]$, where $\alpha \leq n^{c_{9}}$ holds by definition. Then due to $\varepsilon_{n} \geq \frac{1}{n^{c} 8}$ a number of

$$
\left\lceil\frac{2 \cdot \alpha \cdot n^{c_{52}}}{2 \cdot \varepsilon_{n}}\right\rceil \leq n^{c_{54}}
$$

different $\bar{c}_{i, j}^{(r)}$ suffices to guarantee, that at least one of them satisfies the relation (20) for any $c_{i, j}^{(r)}$ with fixed indices. Furthermore, the coefficients $c_{i, j}^{(r)}$, which can actually differ regarding different $h \in \mathcal{H}^{(l)}$, are the ones originating from the coefficients $\mu_{i}, \lambda_{i, l}, \theta_{i, l, v}$ in the definition of $\mathcal{F}_{M^{*}, d^{*}, d, \alpha}^{(\text {enarar network })}$. Using (8), their number can be bounded by $c_{55} \cdot M^{*}$. So the logarithm of the covering number $\mathcal{N}\left(\varepsilon_{n}, \mathcal{H}^{(l)},\|\cdot\|_{\infty,\left[-a_{n}, a_{n}\right]^{d}}\right)$ can be bounded by

$$
\log \left(\mathcal{N}\left(\varepsilon_{n}, \mathcal{H}^{(l)},\|\cdot\|_{\infty,\left[-a_{n}, a_{n}\right]^{d}}\right)\right) \leq \log \left(\left(n^{c_{54}}\right)^{c_{55} \cdot M^{*}}\right) \leq c_{10} \cdot \log (n) \cdot M^{*},
$$

which proves the assertion. 
In the proof of Theorem 2, we will need several auxiliary results, which we formulate and prove next.

For our first auxiliary result we need to introduce the following notations: Let $N \in \mathbb{N}$ and $d \in \mathbb{N}$. For

$$
\alpha=\left(\alpha^{(0)}, \ldots, \alpha^{(d)}\right) \in \mathbb{R}^{d+1}
$$

set

$$
f_{\alpha}(x)=\left(\alpha^{(0)}+\sum_{k=1}^{d} \alpha^{(k)} \cdot x^{(k)}\right)^{N} \quad\left(x \in \mathbb{R}^{d}\right) .
$$

Obviously we have

$$
f_{\alpha}(x)=\sum_{\substack{r_{0}, \ldots, r_{d} \in \mathbb{N}_{0}, r_{0}+\cdots+r_{d}=N}}\left(\begin{array}{c}
N \\
r_{0}, \ldots, r_{d}
\end{array}\right) \cdot \prod_{k=0}^{d}\left(\alpha^{(k)}\right)^{r_{k}} \cdot \prod_{k=1}^{d}\left(x^{(k)}\right)^{r_{k}} .
$$

Let $\mathcal{P}_{N}$ be the linear span of all monomials of the form

$$
\prod_{k=1}^{d}\left(x^{(k)}\right)^{r_{k}}
$$

for some $r_{1}, \ldots, r_{d} \in \mathbb{N}_{0}, r_{1}+\cdots+r_{d} \leq N$. Then, $\mathcal{P}_{N}$ is a linear vector space of functions of dimension

$$
\operatorname{dim} \mathcal{P}_{N}=\left|\left\{\left(r_{0}, \ldots, r_{d}\right) \in \mathbb{N}_{0}^{d+1}: r_{0}+\cdots+r_{d}=N\right\}\right|=\left(\begin{array}{c}
d+N \\
d
\end{array}\right)
$$

and we have $f_{\alpha} \in \mathcal{P}_{N}$ for all $\alpha \in \mathbb{R}^{d+1}$.

Lemma 3. Set $K=\operatorname{dim} \mathcal{P}_{N}$. For almost all $\alpha_{1}, \ldots, \alpha_{K} \in \mathbb{R}^{d+1}$ (with respect to the Lebesgue measure in $\mathbb{R}^{(d+1) \cdot K}$ ) we have that $f_{\alpha_{1}}, \ldots, f_{\alpha_{K}}$ is a basis of the linear vector space $\mathcal{P}_{N}$.

Proof. It suffices to show that $f_{\alpha_{1}}, \ldots, f_{\alpha_{K}}$ are linearly independent. To do this, let $\beta_{1}, \ldots, \beta_{K} \in \mathbb{R}$ be such that

$$
\sum_{k=1}^{K} \beta_{k} \cdot f_{\alpha_{k}}=0
$$

The monomials (22) are linearly independent. Thus, equation (23) implies

$$
\sum_{k=1}^{K} \beta_{k} \cdot \prod_{j=0}^{d}\left(\alpha_{k}^{(j)}\right)^{r_{j}}=0 \quad \text { for all } \mathbf{r}=\left(r_{0}, \ldots, r_{d}\right) \in R
$$

where

$$
R=\left\{\left(r_{0}, \ldots, r_{d}\right) \in \mathbb{N}_{0}^{d+1} \quad: \quad r_{0}+\cdots+r_{d}=N\right\}
$$


It suffices to show that the matrix

$$
A=\left(\prod_{j=0}^{d}\left(\alpha_{k}^{(j)}\right)^{r_{j}}\right)_{\mathbf{r} \in R, k \in\{1, \ldots, K\}}
$$

is regular, which is equivalent to the assertion that the matrix

$$
A^{T}=\left(\prod_{j=0}^{d}\left(\alpha_{k}^{(j)}\right)^{r_{j}}\right)_{k \in\{1, \ldots, K\}, \mathbf{r} \in R}
$$

is regular. To prove this, it suffices to show that for arbitrary $\gamma_{\mathbf{r}} \in \mathbb{R}(\mathbf{r} \in R)$, we have that

$$
\sum_{\mathbf{r} \in R} \gamma_{\mathbf{r}} \cdot \prod_{j=0}^{d}\left(\alpha_{k}^{(j)}\right)^{r_{j}}=0 \quad \text { for } k=1, \ldots, K
$$

implies

$$
\gamma_{\mathbf{r}}=0 \text { for all } \mathbf{r} \in R \text {. }
$$

Thus, let $\gamma_{\mathbf{r}} \in \mathbb{R}(\mathbf{r} \in R)$ be arbitrary and assume that (25) holds. Then the polynomial

$$
p(x)=\sum_{\mathbf{r} \in R} \gamma_{\mathbf{r}} \cdot \prod_{k=1}^{d}\left(x^{(k)}\right)^{r_{k}}
$$

which is contained in $\mathcal{P}_{N}$, satisfies

$$
p\left(\alpha_{k}\right)=0 \text { for } k=1, \ldots, K .
$$

Proposition 4 in Sauer (2006) implies that the condition (27) has the only solution $p=0$ in $\mathcal{P}_{N}$ for Lebesgue almost all $\alpha_{1}, \ldots \alpha_{K} \in \mathbb{R}^{d+1}$, which in turn implies (26). The proof is complete.

Lemma 4. Let $\sigma: \mathbb{R} \rightarrow[0,1]$ satisfy the properties $(i)$ and (ii) of Definition 3. Then for any $R>0$ coefficients $\gamma_{1}, \ldots, \gamma_{N+1} \in \mathbb{R}$ and $\beta_{1}, \ldots, \beta_{N+1}$ with

$$
\left|\gamma_{k}\right| \leq c_{15} \cdot R^{N} \quad \text { and } \quad\left|\beta_{k}\right| \leq \frac{N}{R}
$$

for all $1 \leq k \leq N+1$ exist, such that for all $x \in[-a, a]$

$$
\left|\sum_{k=1}^{N+1} \gamma_{k} \cdot \sigma\left(\beta_{k} \cdot x+t_{\sigma}\right)-x^{N}\right| \leq c_{16} \cdot \frac{a^{N+1}}{R}
$$

holds, where $c_{15}$ and $c_{16}$ depend on $N$ but not on a and $R$. 
Proof. The result follows in a straightforward way from the proof of Theorem 2 in Scarselli and Tsoi (1998). For the sake of completeness we provide nevertheless the detailed proof below.

Since due to Definition 3 there is a point $t_{\sigma} \in \mathbb{R}$, such that none of the derivatives up to the order $N$ is zero in $t_{\sigma}$, the one-layered neural network described in the assertion of this lemma can be formulated explicitly as

$$
\begin{aligned}
& \sum_{k=1}^{N+1}(-1)^{N+k-1} \cdot \frac{R^{N}}{\sigma^{(N)}\left(t_{\sigma}\right)} \cdot\left(\begin{array}{c}
N \\
k-1
\end{array}\right) \cdot \sigma\left(\frac{k-1}{R} \cdot x+t_{\sigma}\right) \\
& =\sum_{k=0}^{N}(-1)^{N+k} \cdot \frac{R^{N}}{\sigma^{(N)}\left(t_{\sigma}\right)} \cdot\left(\begin{array}{c}
N \\
k
\end{array}\right) \cdot \sigma\left(\frac{k}{R} \cdot x+t_{\sigma}\right) \\
& =(-1)^{N} \cdot \frac{R^{N}}{\sigma^{(N)}\left(t_{\sigma}\right)} \cdot \sum_{k=0}^{N}(-1)^{k} \cdot\left(\begin{array}{c}
N \\
k
\end{array}\right) \cdot \sigma\left(\frac{k}{R} \cdot x+t_{\sigma}\right)
\end{aligned}
$$

Since Definition 3 implies, that $\sigma$ is $N+1$ times continously differentiable, it can be expanded in a Taylor series with Lagrange remainder around $t_{\sigma}$ up to order $N$ and we can conclude (defining $0^{0}=1$ )

$$
\begin{aligned}
& \sum_{k=0}^{N}(-1)^{k} \cdot\left(\begin{array}{l}
N \\
k
\end{array}\right) \cdot \sigma\left(\frac{k}{R} \cdot x+t_{\sigma}\right) \\
& =\sum_{k=0}^{N}(-1)^{k} \cdot\left(\begin{array}{l}
N \\
k
\end{array}\right) \cdot\left(\sum_{j=0}^{N} \frac{\sigma^{(j)}\left(t_{\sigma}\right) \cdot(x k)^{j}}{R^{j} \cdot j !}+\frac{\sigma^{(N+1)}\left(\xi_{k}\right) \cdot(x k)^{N+1}}{R^{N+1} \cdot(N+1) !}\right) \\
& =\sum_{j=0}^{N} \frac{\sigma^{(j)}\left(t_{\sigma}\right) \cdot x^{j}}{R^{j} \cdot j !} \cdot \sum_{k=0}^{N}(-1)^{k} \cdot k^{j} \cdot\left(\begin{array}{c}
N \\
k
\end{array}\right) \\
& \quad+\frac{x^{N+1}}{R^{N+1} \cdot(N+1) !} \cdot \sum_{k=0}^{N}(-1)^{k} \cdot k^{N+1} \cdot \sigma^{(N+1)}\left(\xi_{k}\right) \cdot\left(\begin{array}{c}
N \\
k
\end{array}\right),
\end{aligned}
$$

where $\xi_{k} \in\left[t_{\sigma}-\frac{k}{R} \cdot|x|, t_{\sigma}+\frac{k}{R} \cdot|x|\right]$ for all $0 \leq k \leq N$. Next, we notice that

$$
\sum_{k=0}^{N}(-1)^{k} \cdot k^{j} \cdot\left(\begin{array}{l}
N \\
k
\end{array}\right)=N ! \cdot(-1)^{N} \cdot S_{j}^{(N)}
$$

holds, where $S_{j}^{(N)}$ is the well-known Stirling number of the second kind, which describes the number of options to split a set of $j$ elements into $N$ non-empty subsets and which is equal to zero for $0 \leq j<N$ and equal to one for $j=N$ (cf., e.g., the recurrence relation on page 825 in Abramovitz and Stegun (1972), which actually holds for all combinations of $j, N \in \mathbb{N}_{0}$ and implies the mentioned values in connection with the binomial theorem). This simplifies the above sum to

$$
\frac{\sigma^{(N)}\left(t_{\sigma}\right) \cdot x^{N}}{R^{N}} \cdot(-1)^{N}+\frac{x^{N+1}}{R^{N+1} \cdot(N+1) !} \cdot \sum_{k=0}^{N}(-1)^{k} \cdot k^{N+1} \cdot \sigma^{(N+1)}\left(\xi_{k}\right) \cdot\left(\begin{array}{c}
N \\
k
\end{array}\right) .
$$


Plugging this into the representation of (30) leads to

$$
\begin{aligned}
(-1)^{N} \cdot & \frac{R^{N}}{\sigma^{(N)}\left(t_{\sigma}\right)} \cdot\left(\frac{\sigma^{(N)}\left(t_{\sigma}\right) \cdot x^{N}}{R^{N}} \cdot(-1)^{N}\right. \\
& \left.\quad \frac{x^{N+1}}{R^{N+1} \cdot(N+1) !} \cdot \sum_{k=0}^{N}(-1)^{k} \cdot k^{N+1} \cdot \sigma^{(N+1)}\left(\xi_{k}\right) \cdot\left(\begin{array}{c}
N \\
k
\end{array}\right)\right) \\
=x^{N}+ & \frac{(-1)^{N} \cdot x^{N+1}}{R \cdot \sigma^{(N)}\left(t_{\sigma}\right) \cdot(N+1) !} \cdot \sum_{k=0}^{N}(-1)^{k} \cdot k^{N+1} \cdot \sigma^{(N+1)}\left(\xi_{k}\right) \cdot\left(\begin{array}{c}
N \\
k
\end{array}\right) .
\end{aligned}
$$

This implies the assertion of the lemma, since the derivative of order $N+1$ is bounded on $\mathbb{R}$ (cf., Definition 3) and the weights chosen in (29) satisfy the announced bounds.

Lemma 5. Let $p \in \mathcal{P}_{N}$ for $N \in \mathbb{N}_{0}$. Let $m_{1}, \ldots, m_{\left(\begin{array}{c}d+N \\ d\end{array}\right)}$ denote all monomials in $\mathcal{P}_{N}$. Define $r_{i} \in \mathbb{R}\left(i=1, \ldots,\left(\begin{array}{c}d+N \\ d\end{array}\right)\right)$ by

$$
p(x)=\sum_{i=1}^{\left(\begin{array}{c}
d+N \\
d
\end{array}\right)} r_{i} \cdot m_{i}(x) \quad\left(x \in \mathbb{R}^{d}\right),
$$

and set $\bar{r}(p)=\max _{i=1, \ldots,\left(\begin{array}{c}d+N \\ d\end{array}\right)}\left|r_{i}\right|$. Let $\sigma: \mathbb{R} \rightarrow[0,1]$ be $N$-admissible according to Definition 3. Then for any $R, a>0$ a neural network of the type

$$
s(x)=\sum_{j=1}^{\left(\begin{array}{c}
d+N \\
d
\end{array}\right)} d_{j} \sum_{k=1}^{N+1} b_{k} \cdot \sigma\left(\sum_{v=1}^{d} a_{j, k, v} \cdot x^{(v)}+a_{j, k, 0}\right)
$$

exists such that

$$
|s(x)-p(x)| \leq c_{17} \cdot \bar{r}(p) \cdot \frac{a^{N+1}}{R}
$$

holds for all $x \in[-a, a]^{d}$, and the coefficients of this neural network satisfy

$$
\begin{aligned}
& \left|d_{j}\right| \leq c_{18} \cdot \bar{r}(p), \\
& \left|b_{k}\right| \leq c_{15} \cdot R^{N}, \\
& \left|a_{j, k, v}\right| \leq \frac{N \cdot \max \{1, a\}}{R \cdot(d+1)}+\left|t_{\sigma}\right|
\end{aligned}
$$

for $j \in\left\{1, \ldots,\left(\begin{array}{c}d+N \\ d\end{array}\right)\right\}, k \in\{1, \ldots, N+1\}$, and $v \in\{0, \ldots, d\}$, where $c_{18}$ and $c_{15}$ depend on $N$ and $d$ but not on $a, R$ and $p$.

Proof. It follows from Lemma 3 that we can reconstruct all of the $m_{i}$ by a sum of the form

$$
m_{i}(x)=\sum_{j=1}^{\left(\begin{array}{c}
d+N \\
d
\end{array}\right)} \bar{d}_{i, j} \cdot\left(\sum_{v=1}^{d} \alpha_{j}^{(v)} \cdot x^{(v)}+\alpha_{j}^{(0)}\right)^{N}
$$


where we can choose $\alpha_{j}^{(v)} \in\left[-\frac{1}{d+1}, \frac{1}{d+1}\right]$ and $\alpha_{j}^{(0)} \in\left[-\frac{a}{d+1}, \frac{a}{d+1}\right]$ for $j \in\left\{1, \ldots,\left(\begin{array}{c}d+N \\ d\end{array}\right)\right\}$ and $v \in\{1, \ldots, d\}$, because these alternatives form a set with positive Lebesgue measure. Then $\sum_{v=1}^{d} \alpha_{j}^{(v)} \cdot x^{(v)}+\alpha_{j}^{(0)} \in[-a, a]$ holds for all $j \in\left\{1, \ldots,\left(\begin{array}{c}d+N \\ d\end{array}\right)\right\}$. After selecting a possible assignment of this type for all these inner coefficients, we can bound the maximum absolute value of the coefficients $\bar{d}_{i, j}$ by a constant

$$
\max _{i, j \in\left\{1, \ldots,\left(\begin{array}{c}
d+N \\
d
\end{array}\right)\right\}} \bar{d}_{i, j}=c_{19}
$$

If we replace the subfunction $g(z)=z^{N}$ in (32) by its neural network approximation from Lemma 4, we obtain

$$
\begin{aligned}
& \left|m_{i}(x)-\sum_{j=1}^{\left(\begin{array}{c}
d+N \\
d
\end{array}\right)} \bar{d}_{i, j} \cdot\left(\sum_{k=1}^{N+1} \gamma_{k} \cdot \sigma\left(\beta_{k}\left(\sum_{v=1}^{d} \alpha_{j}^{(v)} \cdot x^{(v)}+\alpha_{j}^{(0)}\right)+t_{\sigma}\right)\right)\right| \\
& \leq \sum_{j=1}^{\left(\begin{array}{c}
d+N \\
d
\end{array}\right)}\left|\bar{d}_{i, j}\right| \cdot \mid\left(\sum_{v=1}^{d} \alpha_{j}^{(v)} \cdot x^{(v)}+\alpha_{j}^{(0)}\right)^{N} \\
& \quad-\left(\sum_{k=1}^{N+1} \gamma_{k} \cdot \sigma\left(\sum_{v=1}^{d} \beta_{k} \cdot \alpha_{j}^{(v)} \cdot x^{(v)}+\beta_{k} \cdot \alpha_{j}^{(0)}+t_{\sigma}\right)\right) \mid \\
& \leq\left(\begin{array}{c}
d+N \\
d
\end{array}\right) \cdot c_{19} \cdot c_{16} \cdot \frac{a^{N+1}}{R} .
\end{aligned}
$$

By using representation (31), we conclude

$$
\begin{aligned}
& \mid p(x)-\sum_{i=1}^{\left(\begin{array}{c}
d+N \\
d
\end{array}\right)} r_{i} \sum_{j=1}^{\left(\begin{array}{c}
d+N \\
d
\end{array}\right)} \bar{d}_{i, j} \\
& \quad \cdot\left(\sum_{k=1}^{N+1} \gamma_{k} \cdot \sigma\left(\sum_{v=1}^{d} \beta_{k} \cdot \alpha_{j}^{(v)} \cdot x^{(v)}+\beta_{k} \cdot \alpha_{j}^{(0)}+t_{\sigma}\right)\right) \mid \\
& \leq \sum_{i=1}^{\left(\begin{array}{c}
d+N \\
d
\end{array}\right)}\left|r_{i}\right| \cdot \mid m_{i}(x) \\
& \quad-\sum_{j=1}^{\left(\begin{array}{c}
+N \\
d
\end{array}\right)} \bar{d}_{i, j} \cdot\left(\sum_{k=1}^{N+1} \gamma_{k} \cdot \sigma\left(\sum_{v=1}^{d} \beta_{k} \cdot \alpha_{j}^{(v)} \cdot x^{(v)}+\beta_{k} \cdot \alpha_{j}^{(0)}+t_{\sigma}\right)\right) \mid \\
& \leq\left(\begin{array}{c}
d+N \\
d
\end{array}\right) \cdot \bar{r}(p) \cdot\left(\begin{array}{c}
d+N \\
d
\end{array}\right) \cdot c_{19} \cdot c_{16} \cdot \frac{a^{N+1}}{R} .
\end{aligned}
$$

Thus, $d_{j}=\sum_{i=1}^{\left(\begin{array}{c}d+N \\ d\end{array}\right)} \bar{d}_{i, j} \cdot r_{i}, b_{k}=\gamma_{k}, a_{j, k, v}=\beta_{k} \cdot \alpha_{j}^{(v)}$ and $a_{j, k, 0}=\beta_{k} \cdot \alpha_{j}^{(0)}+t_{\sigma}$ satisfy the assertion of the lemma, because they are bounded in the required way due to (33), 
(28), and the choice of the coefficients subsequent to (32).

Remark 2. We notice that we can rewrite $s(x)$ in Lemma 5 as

$$
s(x)=\sum_{l=1}^{\left(\begin{array}{c}
d+N \\
d
\end{array}\right) \cdot(N+1)} \tilde{b}_{l} \cdot \sigma\left(\sum_{v=1}^{d} \tilde{a}_{l, v} \cdot x^{(v)}+\tilde{a}_{l, 0}\right),
$$

if we define $\tilde{b}_{(j-1) \cdot(N+1)+k}=d_{j} \cdot b_{k}$ and $\tilde{a}_{(j-1) \cdot(N+1)+k, v}=a_{j, k, v}$ for $j=1, \ldots,\left(\begin{array}{c}d+N \\ d\end{array}\right)$ and $k=1, \ldots, N+1$. This allows us to bound these coefficients by

$$
\begin{aligned}
& \left|\tilde{b}_{l}\right| \leq c_{20} \cdot \bar{r}(p) \cdot R^{N}, \\
& \left|\tilde{a}_{l, v}\right| \leq \frac{N \cdot \max \{1, a\}}{R \cdot(d+1)}+\left|t_{\sigma}\right|=c_{21} \cdot \frac{\max \{1, a\}}{R}+\left|t_{\sigma}\right|
\end{aligned}
$$

for all $l=1, \ldots,\left(\begin{array}{c}d+N \\ d\end{array}\right) \cdot(N+1)$ and $v=0, \ldots, d$.

Our next lemma is a modification of Proposition 3.8 in Mhaskar (1993).

Lemma 6. Let $K \subseteq \mathbb{R}^{d}$ be a polytope bounded by hyperplanes $u_{j} \cdot x+w_{j} \leq 0(j=$ $1, \ldots, H)$, where $u_{1}, \ldots, u_{H} \in \mathbb{R}^{d}$ and $w_{1}, \ldots, w_{H} \in \mathbb{R}$. For $\delta>0$ set

$$
K_{\delta}^{0}:=\left\{x \in \mathbb{R}^{d}: u_{j} \cdot x+w_{j} \leq-\delta \quad \text { for all } j \in\{1, \ldots, H\}\right\}
$$

and

$$
K_{\delta}^{c}:=\left\{x \in \mathbb{R}^{d}: u_{j} \cdot x+w_{j} \geq \delta \quad \text { for some } j \in\{1, \ldots, H\}\right\} .
$$

Let $\sigma: \mathbb{R} \rightarrow[0,1]$ be a sigmoidal function, which satisfies

$$
|\sigma(y)-1| \leq \frac{1}{y} \quad \text { if } \quad y>0 \quad \text { and } \quad|\sigma(y)| \leq \frac{1}{|y|} \quad \text { if } \quad y<0 .
$$

Let $\varepsilon, \delta \in(0,1]$ be arbitrary. Then a neural network of the form

$$
f(x)=\sum_{j=1}^{H} \sigma\left(\sum_{k=1}^{d} a_{j, k} \cdot x^{(k)}+a_{j, 0}\right)
$$

exists, satisfying

$$
\begin{aligned}
& |f(x)| \leq H \text { for } x \in \mathbb{R}^{d}, \\
& |f(x)| \leq H \cdot \varepsilon \text { for } x \in K_{\delta}^{0}, \\
& f(x) \geq 1-\varepsilon \text { for } x \in K_{\delta}^{c} .
\end{aligned}
$$

The weights above can be chosen such that

$$
\left|a_{j, k}\right| \leq \frac{1}{\varepsilon \cdot \delta} \cdot \max \left\{\left\|u_{1}\right\|_{\infty},\left|w_{1}\right|, \ldots,\left\|u_{H}\right\|_{\infty},\left|w_{H}\right|\right\}
$$

for all $j=1, \ldots, H$ and $k=0, \ldots, d$. 
Proof. We set

$$
a_{j, k}=\frac{1}{\varepsilon \cdot \delta} \cdot u_{j}^{(k)} \quad \text { and } \quad a_{j, 0}=\frac{1}{\varepsilon \cdot \delta} \cdot w_{j} \quad \text { for all } j=1, \ldots, H ; k=1, \ldots, d .
$$

So for $x \in K_{\delta}^{0}$

$$
\sum_{k=1}^{d} a_{j, k} \cdot x^{(k)}+a_{j, 0} \leq-\frac{1}{\varepsilon} \text { for all } j=1, \ldots, H,
$$

which implies

$$
\left|\sum_{j=1}^{H} \sigma\left(\sum_{k=1}^{d} a_{j, k} \cdot x^{(k)}+a_{j, 0}\right)\right| \leq \sum_{j=1}^{H}\left|\sigma\left(\sum_{k=1}^{d} a_{j, k} \cdot x^{(k)}+a_{j, 0}\right)\right| \leq H \cdot \varepsilon
$$

due to (34). For $x \in K_{\delta}^{c}$ we know that there is a $j^{*} \in\{1, \ldots, H\}$, which satisfies

$$
\sum_{k=1}^{d} a_{j^{*}, k} \cdot x^{(k)}+a_{j^{*}, 0} \geq \frac{1}{\varepsilon}
$$

This leads to

$$
\sum_{j=1}^{H} \sigma\left(\sum_{k=1}^{d} a_{j, k} \cdot x^{(k)}+a_{j, 0}\right) \geq \sigma\left(\sum_{k=1}^{d} a_{j^{*}, k} \cdot x^{(k)}+a_{j^{*}, 0}\right) \geq 1-\varepsilon
$$

because of (34) and $\sigma(y) \geq 0$ for all $y \in \mathbb{R}$. Furthermore, $\|\sigma\|_{\infty} \leq 1$ implies $|f(x)| \leq H$ and the announced bound for the coefficients follows immediately from their definition above.

Lemma 7. Let $K \subseteq \mathbb{R}^{d}$ be a polytope bounded by hyperplanes $v_{j} \cdot x+w_{j} \leq 0 \quad(j=$ $1, \ldots, H)$, where $v_{1}, \ldots, v_{H} \in \mathbb{R}^{d}$ and $w_{1}, \ldots, w_{H} \in \mathbb{R}$, and let $a \geq 1$. Let $M \in \mathbb{N}$ be sufficiently large (independent of the size of $a$, but $a \leq M$ must hold). For $\delta>0$ define $K_{\delta}^{0}$ and $K_{\delta}^{c}$ as in Lemma 6 . Let $p: \mathbb{R}^{d} \rightarrow \mathbb{R}$ be a polynomial from $\mathcal{P}_{N}$ with $\bar{r}(p)$ defined as in Lemma 5 and let $\sigma: \mathbb{R} \rightarrow[0,1]$ be $N$-admissible according to Definition 3. Then a function

$$
t(x)=\sum_{j=1}^{\left(\begin{array}{c}
d+N \\
d
\end{array}\right) \cdot(N+1)} \mu_{j} \cdot \sigma\left(\sum_{l=1}^{2 d+H} \lambda_{j, l} \cdot \sigma\left(\sum_{v=1}^{d} \theta_{l, v} \cdot x^{(v)}+\theta_{l, 0}\right)+\lambda_{j, 0}\right)
$$

exists, such that

$$
\begin{aligned}
|t(x)-p(x)| & \leq c_{22} \cdot \bar{r}(p) \cdot a^{N+3} \cdot M^{-p} & & \text { for } x \in K_{\delta}^{0} \cap[-a, a]^{d}, \\
|t(x)| & \leq c_{23} \cdot \bar{r}(p) \cdot M^{-d-2 p} & & \text { for } x \in K_{\delta}^{c} \cap[-a, a]^{d},
\end{aligned}
$$




$$
|t(x)| \leq c_{24} \cdot \bar{r}(p) \cdot M^{N \cdot p}
$$

for $x \in \mathbb{R}^{d}$

hold. Here the coefficients can be chosen such that they satisfy

$$
\begin{aligned}
\left|\mu_{j}\right| & \leq c_{20} \cdot \bar{r}(p) \cdot M^{N \cdot p}, \\
\left|\lambda_{j, l}\right| & \leq M^{d+p \cdot(N+2)}, \\
\left|\theta_{l, v}\right| & \leq \max \left\{\left|t_{\sigma}\right|, \frac{M^{d+p \cdot(2 N+3)}}{\delta} \cdot \max \left\{\left\|u_{1}\right\|_{\infty},\left|w_{1}\right|, \ldots,\left\|u_{H}\right\|_{\infty},\left|w_{H}\right|\right\}\right\},
\end{aligned}
$$

for $j \in\left\{1, \ldots,\left(\begin{array}{c}d+N \\ d\end{array}\right) \cdot(N+1)\right\}, l \in\{0, \ldots, 2 d+H\}$, and $v \in\{0, \ldots, d\}$.

Proof. Let $t_{\sigma}, c_{21}$ and $c_{15}$ be defined as in Remark 2 and Lemma 4 and set $R=M^{p}$, $\tilde{R}=M^{p \cdot(N+1)}, B=M^{d+p \cdot(N+2)}, \varepsilon=M^{-d-p \cdot(2 N+3)}$. For a sufficiently large $M \in \mathbb{N}$, we have

$$
\begin{aligned}
& \left(c_{21} \cdot \frac{a}{M^{p}}+\left|t_{\sigma}\right|\right) \cdot\left(2 \cdot c_{15} \cdot M^{p \cdot(N+1)}+1\right) \\
& \leq M^{d+p \cdot(N+2)} \cdot\left(\frac{3}{4}-M^{-d-p \cdot(2 N+3)}\right)
\end{aligned}
$$

because $a \leq M$. Consequently $R, \tilde{R}, B$, and $\varepsilon$ satisfy

$$
\left(c_{21} \cdot \frac{a}{R}+\left|t_{\sigma}\right|\right) \cdot\left(2 \cdot c_{15} \cdot \tilde{R}+1\right) \leq B \cdot\left(\frac{3}{4}-\varepsilon\right) .
$$

Let

$$
s(x)=\sum_{j=1}^{\left(\begin{array}{c}
d+N \\
d
\end{array}\right) \cdot(N+1)} \tilde{b}_{j} \cdot \sigma\left(\sum_{l=1}^{d} \tilde{a}_{j, l} \cdot x^{(l)}+\tilde{a}_{j, 0}\right),
$$

be chosen as the approximation in Remark 2 using the above $R$. At first, we replace the terms $x^{(l)}$ by their approximation from Lemma 4 using $N=1$ and $\tilde{R}$ therein and insert an additional term of the type $f(x)$ in Lemma 6 multiplied by $-B$. This leads to

$$
\begin{aligned}
t(x)=\sum_{j=1}^{\left(\begin{array}{c}
d+N \\
d
\end{array}\right) \cdot(N+1)} \tilde{b}_{j} & \cdot \sigma\left(\sum_{l=1}^{d} \tilde{a}_{j, l} \cdot \sum_{k=1}^{2} \gamma_{k} \cdot \sigma\left(\beta_{k} \cdot x^{(l)}+t_{\sigma}\right)\right. \\
& \left.-B \cdot \sum_{l=1}^{H} \sigma\left(\sum_{k=1}^{d} a_{l, k} \cdot x^{(k)}+a_{l, 0}\right)+\tilde{a}_{j, 0}\right) .
\end{aligned}
$$

Since the properties of $\sigma$ entail Lipschitz continuity with a Lipschitz constant $L>0$, Lemma 4, Lemma 6 respecting the above $\varepsilon$, and Remark 2 imply for $x \in K_{\delta}^{0} \cap[-a, a]^{d}$

$$
|t(x)-p(x)|
$$




$$
\begin{aligned}
& \leq\left|t(x)-\sum_{j=1}^{\left(\begin{array}{c}
d+N \\
d
\end{array}\right) \cdot(N+1)} \tilde{b}_{j} \cdot \sigma\left(\sum_{l=1}^{d} \tilde{a}_{j, l} \cdot \sum_{k=1}^{2} \gamma_{k} \cdot \sigma\left(\beta_{k} \cdot x^{(l)}+t_{\sigma}\right)+\tilde{a}_{j, 0}\right)\right| \\
& +\left|\sum_{j=1}^{\left(\begin{array}{c}
d+N \\
d
\end{array}\right) \cdot(N+1)} \tilde{b}_{j} \cdot \sigma\left(\sum_{l=1}^{d} \tilde{a}_{j, l} \cdot \sum_{k=1}^{2} \gamma_{k} \cdot \sigma\left(\beta_{k} \cdot x^{(l)}+t_{\sigma}\right)+\tilde{a}_{j, 0}\right)-s(x)\right| \\
& +|s(x)-p(x)| \\
& \leq \sum_{j=1}^{\left(\begin{array}{c}
d+N \\
d
\end{array}\right) \cdot(N+1)}\left|\tilde{b}_{j}\right| \cdot L \cdot B \cdot\left|\sum_{l=1}^{H} \sigma\left(\sum_{k=1}^{d} a_{l, k} \cdot x^{(k)}+a_{l, 0}\right)\right| \\
& +\sum_{j=1}^{\left(\begin{array}{c}
d+N \\
d
\end{array}\right) \cdot(N+1)}\left|\tilde{b}_{j}\right| \cdot L \cdot \sum_{l=1}^{d}\left|\tilde{a}_{j, l}\right| \cdot\left|\sum_{k=1}^{2} \gamma_{k} \cdot \sigma\left(\beta_{k} \cdot x^{(l)}+t_{\sigma}\right)-x^{(l)}\right| \\
& +|s(x)-p(x)| \\
& \leq\left(\begin{array}{c}
d+N \\
d
\end{array}\right) \cdot(N+1) \cdot c_{20} \cdot \bar{r}(p) \cdot R^{N} \cdot L \cdot B \cdot H \cdot \varepsilon \\
& +\left(\begin{array}{c}
d+N \\
d
\end{array}\right) \cdot(N+1) \cdot c_{20} \cdot \bar{r}(p) \cdot R^{N} \cdot L \cdot d \cdot\left(c_{21} \cdot \frac{a}{R}+\left|t_{\sigma}\right|\right) \cdot c_{16} \cdot \frac{a^{2}}{\tilde{R}} \\
& +c_{17} \cdot \bar{r}(p) \cdot \frac{a^{N+1}}{R} \\
& \leq c_{25} \cdot \bar{r}(p) \cdot\left(R^{N} \cdot B \cdot \varepsilon+\left(\frac{a}{R}+\left|t_{\sigma}\right|\right) \cdot R^{N} \cdot \frac{a^{2}}{\tilde{R}}+\frac{a^{N+1}}{R}\right) \\
& \leq c_{22} \cdot \bar{r}\left(p_{\mathbf{i}}\right) \cdot a^{N+3} \cdot M^{-p} .
\end{aligned}
$$

For $x \in K_{\delta}^{c} \cap[-a, a]^{d}$ we know for the same reason and from the monotonicity of $\sigma$ that

$$
\begin{aligned}
&|t(x)| \leq \sum_{j=1}^{\left(\begin{array}{c}
d+N \\
d
\end{array}\right) \cdot(N+1)}\left|\tilde{b}_{j}\right| \cdot \sigma\left(\sum_{l=1}^{d} \tilde{a}_{j, l} \cdot \sum_{k=1}^{2} \gamma_{k} \cdot \sigma\left(\beta_{k} \cdot x^{(l)}+t_{\sigma}\right)\right. \\
&\left.-B \cdot \sum_{l=1}^{H} \sigma\left(\sum_{k=1}^{d} a_{l, k} \cdot x^{(k)}+a_{l, 0}\right)+\tilde{a}_{j, 0}\right) \\
& \leq \sum_{j=1}^{\left(\begin{array}{c}
d+N \\
d
\end{array}\right) \cdot(N+1)}\left|\tilde{b}_{j}\right| \cdot \sigma\left(\left(c_{21} \cdot \frac{a}{R}+\left|t_{\sigma}\right|\right) \cdot 2 \cdot c_{15} \cdot \tilde{R} \cdot 1\right. \\
& \leq\left.-B \cdot(1-\varepsilon)+c_{21} \cdot \frac{a}{R}+\left|t_{\sigma}\right|\right) \\
& \leq\left(\begin{array}{c}
d+N \\
d=1 \\
d+N
\end{array}\right) \cdot(N+1) \\
& d
\end{aligned}
$$




$$
\leq c_{23} \cdot \bar{r}(p) \cdot \frac{R^{N}}{B} \leq c_{23} \cdot \bar{r}(p) \cdot M^{-d-2 p},
$$

where (36) and property (iii) in Definition 3 were used in the third and fourth inequality, respectively. Moreover, the property $\|\sigma\|_{\infty} \leq 1$ implies

$$
\begin{aligned}
|t(x)| & \leq \sum_{j=1}^{\left(\begin{array}{c}
d+N \\
d
\end{array}\right) \cdot(N+1)}\left|\tilde{b}_{j}\right| \cdot 1 \leq\left(\begin{array}{c}
d+N \\
d
\end{array}\right) \cdot(N+1) \cdot c_{20} \cdot \bar{r}(p) \cdot R^{N} \\
& \leq c_{24} \cdot \bar{r}(p) \cdot R^{N} \leq c_{24} \cdot \bar{r}(p) \cdot M^{N \cdot p}
\end{aligned}
$$

for all $x \in \mathbb{R}^{d}$.

Next we observe that we can condense the representation of $t(x)$ in (37) into

$$
\begin{aligned}
t(x)= & \sum_{j=1}^{\left(\begin{array}{c}
d+N \\
d
\end{array}\right) \cdot(N+1)} \mu_{j} \cdot \sigma\left(\sum_{l=1}^{2 d} \lambda_{j, l} \cdot \sigma\left(\sum_{v=1}^{d} \theta_{l, v} \cdot x^{(v)}+\theta_{l, 0}\right)\right. \\
& \left.\quad+\sum_{l=2 d+1}^{2 d+H} \lambda_{j, l} \cdot \sigma\left(\sum_{v=1}^{d} \theta_{l, v} \cdot x^{(v)}+\theta_{l, 0}\right)+\lambda_{j, 0}\right) \\
= & \sum_{j=1}^{\left({ }^{+N}\right) \cdot(N+1)} \mu_{j} \cdot \sigma\left(\sum_{l=1}^{2 d+H} \lambda_{j, l} \cdot \sigma\left(\sum_{v=1}^{d} \theta_{l, v} \cdot x^{(v)}+\theta_{l, 0}\right)+\lambda_{j, 0}\right),
\end{aligned}
$$

if we choose

$$
\begin{aligned}
\mu_{j} & =\tilde{b}_{j} \\
\lambda_{j, l} & = \begin{cases}\tilde{a}_{j, 0} & \text { if } l=0 \\
\tilde{a}_{j,\left\lceil\frac{l}{2}\right\rceil} \cdot \gamma_{2-l+2 \cdot\left\lfloor\frac{l}{2}\right\rfloor} & \text { if } l \in\{1, \ldots, 2 d\} \\
-B & \text { if } l \in\{2 d+1, \ldots, 2 d+H\}\end{cases} \\
\theta_{l, v} & = \begin{cases}t_{\sigma} & \text { if } l \in\{1, \ldots, 2 d\}, v=0 \\
\beta_{2-l+2 \cdot\left\lfloor\frac{l}{2}\right\rfloor} \cdot 1_{\left\{\left\lceil\frac{l}{2}\right\rceil=v\right\}} & \text { if } l \in\{1, \ldots, 2 d\}, v \in\{1, \ldots, d\} . \\
a_{l-2 d, v} & \text { if } l \in\{2 d+1, \ldots, 2 d+H\}\end{cases}
\end{aligned}
$$

For sufficiently large $M$, this leads to

$$
\begin{aligned}
\left|\mu_{j}\right| & \leq c_{20} \cdot \bar{r}(p) \cdot R^{N}=c_{20} \cdot \bar{r}(p) \cdot M^{N \cdot p}, \\
\left|\lambda_{j, l}\right| & \leq \max \left\{c_{21} \cdot \frac{a}{R}+\left|t_{\sigma}\right|,\left(c_{21} \cdot \frac{a}{R}+\left|t_{\sigma}\right|\right) \cdot c_{15} \cdot \tilde{R}, B\right\}=M^{d+p \cdot(N+2)}, \\
\left|\theta_{l, v}\right| & \leq \max \left\{\left|t_{\sigma}\right|, \frac{1}{\tilde{R}}, \frac{1}{\varepsilon \cdot \delta} \cdot \max \left\{\left\|u_{1}\right\|_{\infty},\left|w_{1}\right|, \ldots,\left\|u_{H}\right\|_{\infty},\left|w_{H}\right|\right\}\right\} \\
& =\max \left\{\left|t_{\sigma}\right|, \frac{M^{d+p \cdot(2 N+3)}}{\delta} \cdot \max \left\{\left\|u_{1}\right\|_{\infty},\left|w_{1}\right|, \ldots,\left\|u_{H}\right\|_{\infty},\left|w_{H}\right|\right\}\right\},
\end{aligned}
$$


which completes the proof.

Lemma 8. Let $p=q+s$ for some $q \in \mathbb{N}_{0}$ and $s \in(0,1]$, and let $C>0$. Let $f: \mathbb{R}^{d} \rightarrow \mathbb{R}$ be a $(p, C)$-smooth function, let $x_{0} \in \mathbb{R}^{d}$ and let $p_{q}$ be the Taylor polynomial of total degree $q$ around $x_{0}$, i.e.,

$$
\begin{aligned}
p_{q}(x)= & \sum_{\substack{j_{1}, \ldots, j_{d} \in\{0,1, \ldots, q\} \\
j_{1}+\cdots+j_{d} \leq q}} \frac{1}{j_{1} ! \cdots j_{d} !} \cdot \frac{\partial^{j_{1}+\cdots+j_{d}} f}{\partial^{j_{1}} x^{(1)} \cdots \partial^{j_{d}} x^{(d)}}\left(x_{0}\right) \\
& \cdot\left(x^{(1)}-x_{0}^{(1)}\right)^{j_{1}} \cdots\left(x^{(d)}-x_{0}^{(d)}\right)^{j_{d}} .
\end{aligned}
$$

Then for any $x \in \mathbb{R}^{d}$

$$
\left|f(x)-p_{q}(x)\right| \leq c_{26} \cdot C \cdot\left\|x-x_{0}\right\|^{p}
$$

holds for some constant $c_{26}>0$ depending only on $q$ and $d$.

Proof. See Lemma 1 in Kohler (2014).

Proof of Theorem 2. We subdivide $\left[-a-\frac{2 a}{M}, a\right]^{d}$ into $(M+1)^{d}$ cubes of side length $2 a / M$ and for comprehensibility, we number these cubes $C_{\mathbf{i}}$ by $\mathbf{i} \in\{1, \ldots, M+1\}^{d}$, such that index $\mathbf{i}=\left(i_{1}, \ldots, i_{d}\right)$ corresponds to the cube

$$
\begin{aligned}
& {\left[-a+\left(i_{1}-2\right) \cdot \frac{2 a}{M},-a+\left(i_{1}-1\right) \cdot \frac{2 a}{M}\right] \times \cdots} \\
& \cdots \times\left[-a+\left(i_{d}-2\right) \cdot \frac{2 a}{M},-a+\left(i_{d}-1\right) \cdot \frac{2 a}{M}\right] .
\end{aligned}
$$

Moreover, we denote the corners of these cubes by $x_{\mathbf{i}}$ for $\mathbf{i} \in\{1, \ldots, M+2\}^{d}$ in the same way, such that for all $C_{\mathbf{i}}$ the point $x_{\mathbf{i}}$ means the "bottom left" corner of this cube and the additional indices result from the right border of the whole grid. Therefore, each cube $C_{\mathbf{i}}$ can be written as a polytope defined by

$$
-x^{(j)}+x_{\mathbf{i}}^{(j)} \leq 0 \quad \text { and } \quad x^{(j)}-x_{\mathbf{i}}^{(j)}-\frac{2 a}{M}=x^{(j)}-x_{\mathbf{i}+\mathbf{1}}^{(j)} \leq 0 \quad(j=1, \ldots, d),
$$

where $\mathbf{i}+\mathbf{1}$ means that each component of $\mathbf{i}$ is increased by 1 .

Let $p_{\mathbf{i}}$ denote the Taylor polynomial of $m$ with order $q$ around the center of $C_{\mathbf{i}}$. For each $\mathbf{i} \in\{1, \ldots, M+1\}^{d}$, we treat $C_{\mathbf{i}}$ as $K$ in Lemma 7 . This implies $H=2 d$ therein and we choose $N \in \mathbb{N}_{0}$ with $N \geq q$ and $\delta=a \cdot \eta /(2 \cdot d \cdot M)$. Lemma 7 says that for a sufficiently large $M$ neural networks $t_{\mathbf{i}}(x)$ of the type

$$
\begin{aligned}
t_{\mathbf{i}}(x)= & \sum_{j=1}^{\left(\begin{array}{c}
d+N \\
d
\end{array}\right) \cdot(N+1)}\left(\mu_{j}\right)_{\mathbf{i}} \cdot \sigma\left(\sum _ { l = 1 } ^ { 4 d } ( \lambda _ { j , l } ) _ { \mathbf { i } } \cdot \sigma \left(\sum_{v=1}^{d}\left(\theta_{l, v}\right)_{\mathbf{i}} \cdot x^{(v)}\right.\right. \\
& \left.\left.+\left(\theta_{l, 0}\right)_{\mathbf{i}}\right)+\left(\lambda_{j, 0}\right)_{\mathbf{i}}\right)
\end{aligned}
$$


exist, with coefficients bounded as therein, such that

$$
\begin{aligned}
\left|t_{\mathbf{i}}(x)-p_{\mathbf{i}}(x)\right| & \leq c_{22} \cdot \bar{r}\left(p_{\mathbf{i}}\right) \cdot a^{N+3} \cdot M^{-p} & & \text { for } x \in\left(C_{\mathbf{i}}\right)_{\delta}^{0} \cap[-a, a]^{d}, \\
\left|t_{\mathbf{i}}(x)\right| & \leq c_{23} \cdot \bar{r}\left(p_{\mathbf{i}}\right) \cdot M^{-d-2 p} & & \text { for } x \in\left(C_{\mathbf{i}}\right)_{\delta}^{c} \cap[-a, a]^{d}, \\
\left|t_{\mathbf{i}}(x)\right| & \leq c_{24} \cdot \bar{r}\left(p_{\mathbf{i}}\right) \cdot M^{N \cdot p} & & \text { for } x \in \mathbb{R}^{d}
\end{aligned}
$$

hold for the corresponding cube with index $\mathbf{i} \in\{1, \ldots, M+1\}^{d}$. Assumption (14) in Theorem 2 and a transformation of the Taylor polynomial

$$
\begin{aligned}
p_{\mathbf{i}}(x)= & \sum_{\substack{j_{1}, \ldots, j_{d} \in\{0,1, \ldots, q\} \\
j_{1}+\cdots+j_{d} \leq q}} \frac{1}{j_{1} ! \cdots j_{d} !} \cdot \frac{\partial^{j_{1}+\cdots+j_{d}} m}{\partial^{j_{1}} x^{(1)} \cdots \partial^{j_{d}} x^{(d)}}\left(x_{\mathbf{i}}\right) \\
& \cdot\left(x^{(1)}-x_{\mathbf{i}}^{(1)}\right)^{j_{1}} \cdots\left(x^{(d)}-x_{\mathbf{i}}^{(d)}\right)^{j_{d}}
\end{aligned}
$$

(cf., Lemma 8) into a representation with monomials allow us to bound all values $\bar{r}\left(p_{\mathbf{i}}\right)$ by

$$
\max _{\mathbf{i} \in\{1, \ldots, M+1\}^{d}} \bar{r}\left(p_{\mathbf{i}}\right) \leq c_{27} \cdot a^{q}
$$

for a constant $c_{27}>0$ which depends on $q$ but not on $a$. Set

$$
t(x)=\sum_{\mathbf{i} \in\{1, \ldots, M+1\}^{d}} t_{\mathbf{i}}(x) .
$$

Then, we obtain (from the inequalities above and Lemma 8) for $x \in\left(C_{\mathbf{i}}\right)_{\delta}^{0} \cap[-a, a]^{d}$

$$
\begin{aligned}
|t(x)-m(x)| \leq & \left|t_{\mathbf{i}}(x)-p_{\mathbf{i}}(x)\right|+\left|p_{\mathbf{i}}(x)-m(x)\right|+\left|\sum_{\mathbf{j} \in\{1, \ldots, M+1\}^{d} \backslash\{\mathbf{i}\}} t_{\mathbf{j}}(x)\right| \\
\leq & c_{22} \cdot \bar{r}\left(p_{\mathbf{i}}\right) \cdot a^{N+3} \cdot M^{-p}+c_{26} \cdot C \cdot d^{\frac{p}{2}} \cdot\left(\frac{a}{M}\right)^{p} \\
& +\left((M+1)^{d}-1\right) \cdot c_{23} \cdot \bar{r}\left(p_{\mathbf{i}}\right) \cdot M^{-d-2 p} \\
\leq & c_{13} \cdot a^{N+q+3} \cdot M^{-p} .
\end{aligned}
$$

Arguing in the same way for all $\mathbf{i} \in\{1, \ldots, M+1\}^{d}$ we can conclude that this bound holds for all $x \in[-a, a]^{d}$ which are not contained in

$$
\bigcup_{j=1, \ldots, d \mathbf{i} \in\{1, \ldots, M+2\}^{d}}\left\{x \in \mathbb{R}^{d}:\left|x^{(j)}-x_{\mathbf{i}}^{(j)}\right|<\delta\right\} .
$$

By slightly shifting the whole grid of cubes along the $j$ th component (i.e. modifying all $x_{\mathbf{i}}^{(j)}$ by the same additional summand which is less than $\left.\frac{2 a}{M}\right)$ for fixed $j \in\{1, \ldots, d\}$ we can construct

$$
\left\lfloor\frac{2 a / M}{2 \delta}\right\rfloor=\left\lfloor\frac{2 a}{M} \cdot \frac{2 \cdot d \cdot M}{2 \cdot a \cdot \eta}\right\rfloor=\left\lfloor\frac{2 \cdot d}{\eta}\right\rfloor \geq d / \eta
$$


different versions of $\mathrm{t}$, that still satisfy (40) for all $x \in[-a, a]^{d}$ up to corresponding disjoint versions of

$$
\bigcup_{\mathbf{i} \in\{1, \ldots, M+2\}^{d}}\left\{x \in \mathbb{R}^{d}:\left|x^{(j)}-x_{\mathbf{i}}^{(j)}\right|<\delta\right\},
$$

within (41), and because the sum of the $\nu$-measures of these sets is less than or equal to one, at least one of them must have measure less than or equal to $\eta / d$. Consequently we can shift the $x_{\mathbf{i}}$ such that (41) has $\nu$-measure less than or equal to $\eta$. This finding implies the first assertion of the theorem.

Furthermore, we can bound the coefficients of $t(x)$, if we use the bounds provided by Lemma 7 and observe that due to (38) in this case

$$
u_{j} \in\left\{-\mathbf{e}_{v}, \mathbf{e}_{v}: v \in\{1, \ldots, d\}\right\}
$$

(where $\mathbf{e}_{v}$ denotes the $v$ th unit vector) and

$$
w_{j} \in\left\{x_{\mathbf{i}}^{(v)},-x_{\mathbf{i}+\mathbf{1}}^{(v)}: v \in\{1, \ldots, d\}\right\}
$$

hold for each $C_{\mathbf{i}}$ and $j=1, \ldots, 2 d$. An easy computation shows that the weights satisfy the announced bounds, which completes the proof.

Proof of Theorem 3. We will prove the result by induction and ignore the case $c \cdot \eta_{n} \geq 1$, which is trivially true. For a function $m(x)=f\left(a_{1}^{T} x, \ldots, a_{d^{*}}^{T} x\right)$, which satisfies a generalized hierarchical interaction model of order $d^{*}$ and level $l=0$, let $s: \mathbb{R}^{d} \rightarrow \mathbb{R}^{d^{*}}$ be characterized by $s(x)=\left(a_{1}^{T} x, \ldots, a_{d^{*}}^{T} x\right)^{T}$ and let $\bar{a}_{\max }$ denote $\max _{k=1, \ldots, d^{*}}\left\|a_{k}\right\|_{\infty}$. Applying Theorem 2 (which is possible because of the assumptions of this theorem) for the probability measure $\mathbf{P}_{s(X)}$, the function $f: \mathbb{R}^{d^{*}} \rightarrow \mathbb{R}$ in $m$ can be approximated by a two-layered neural network $\hat{f}$ for all $x \in\left[-d \cdot \bar{a}_{\max } \cdot a_{n}, d \cdot \bar{a}_{\max } \cdot a_{n}\right]^{d^{*}}$, except for a set $\tilde{D}_{0}$ of $\mathbf{P}_{s(X)}$-measure less than or equal to $c \cdot \eta_{n}>0$, with an error of

$$
|\hat{f}(x)-f(x)| \leq c_{13} \cdot\left(d \cdot \bar{a}_{\max } \cdot a_{n}\right)^{N+q+3} \cdot M_{n}^{-p} \leq c_{29} \cdot a_{n}^{N+q+3} \cdot M_{n}^{-p} .
$$

If we plug $s(x)$ into that approximation and condense the inner coefficients per summand, this leads (using the notation of Theorem 2) to the approximation $t(x)=\hat{f}(s(x))$ of the form

$$
\begin{aligned}
t(x) & =\sum_{i=1}^{\left(\begin{array}{c}
d^{*}+N \\
d^{*}
\end{array}\right) \cdot(N+1) \cdot\left(M_{n}+1\right)^{d^{*}}} \mu_{i} \cdot \sigma\left(\sum_{l=1}^{4 d^{*}} \lambda_{i, l} \cdot \sigma\left(\sum_{k=1}^{d^{*}} \theta_{i, l, k} \cdot a_{k}^{T} x+\theta_{i, l, 0}\right)+\lambda_{i, 0}\right) \\
& =\sum_{i=1}^{\left(\begin{array}{c}
d^{*}+N \\
d^{*}
\end{array}\right) \cdot(N+1) \cdot\left(M_{n}+1\right)^{d^{*}}} \mu_{i} \cdot \sigma\left(\sum_{l=1}^{4 d^{*}} \lambda_{i, l} \cdot \sigma\left(\sum_{v=1}^{d} \sum_{k=1}^{d^{*}} a_{k}^{(v)} \cdot \theta_{i, l, k} \cdot x^{(v)}+\theta_{i, l, 0}\right)+\lambda_{i, 0}\right) \\
& =\sum_{i=1}^{\left(\begin{array}{c}
d^{*}+N \\
d^{*}
\end{array}\right) \cdot(N+1) \cdot\left(M_{n}+1\right)^{d^{*}}} \mu_{i} \cdot \sigma\left(\sum_{l=1}^{4 d^{*}} \lambda_{i, l} \cdot \sigma\left(\sum_{v=1}^{d} \tilde{\theta}_{i, l, v} \cdot x^{(v)}+\theta_{i, l, 0}\right)+\lambda_{i, 0}\right),
\end{aligned}
$$


where

$$
\begin{aligned}
\left|\mu_{i}\right| & \leq c_{14} \cdot\left(d \cdot \bar{a}_{\max } \cdot a_{n}\right)^{q} \cdot M_{n}^{N \cdot p} \leq \alpha, \\
\left|\lambda_{i, l}\right| & \leq M_{n}^{d^{*}+p \cdot(N+2)} \leq \alpha, \\
\left|\tilde{\theta}_{i, l, v}\right| & \leq d^{*} \cdot \bar{a}_{\max } \cdot 6 \cdot d^{*} \cdot \frac{1}{\eta_{n}} \cdot M_{n}^{d^{*}+p \cdot(2 N+3)+1} \leq \alpha
\end{aligned}
$$

are satisfied for a sufficiently large $n$, such that $t \in \mathcal{H}^{(0)}$ is valid. Since $\mathbf{P}_{s(X)}\left\{\tilde{D}_{0}\right\}=$ $\mathbf{P}_{X}\left\{s^{-1}\left(\tilde{D}_{0}\right)\right\}$ and $s\left(\left[-a_{n}, a_{n}\right]^{d}\right) \subseteq\left[-d \cdot \bar{a}_{\max } \cdot a_{n}, d \cdot \bar{a}_{\max } \cdot a_{n}\right]^{d^{*}}$,

$$
|t(x)-m(x)| \leq c_{29} \cdot a_{n}^{N+q+3} \cdot M_{n}^{-p}
$$

holds for all $x \in\left[-a_{n}, a_{n}\right]^{d}$ outside of the set $D_{0}=s^{-1}\left(\tilde{D}_{0}\right)$ of $\mathbf{P}_{X}$-measure less than or equal to $c \cdot \eta_{n}$, which proves the first part of the assertion for $l=0$. Furthermore, since $\|\sigma\|_{\infty} \leq 1$ holds according to our assumptions, we know that

$$
\begin{aligned}
|t(x)| & \leq\left(\begin{array}{c}
d^{*}+N \\
d^{*}
\end{array}\right) \cdot(N+1) \cdot\left(M_{n}+1\right)^{d^{*}} \cdot \max _{i=1, \ldots,\left(\begin{array}{c}
d^{*}+N \\
d^{*}
\end{array}\right) \cdot(N+1) \cdot\left(M_{n}+1\right)^{d^{*}}}\left|\mu_{i}\right| \\
& \leq c_{31} \cdot a_{n}^{q} \cdot M_{n}^{d^{*}+N \cdot p}
\end{aligned}
$$

is valid for all $x \in \mathbb{R}^{d}$.

When $l>0$, we consider the following bound of the difference between the function $m(x)=\sum_{k=1}^{K} g_{k}\left(f_{1, k}(x), \ldots, f_{d^{*}, k}(x)\right)$ and an estimate with the structure $\hat{m}(x)=$ $\sum_{k=1}^{K} \hat{g}_{k}\left(\hat{f}_{1, k}(x), \ldots, \hat{f}_{d^{*}, k}(x)\right)$ at a point $x \in\left[-a_{n}, a_{n}\right]^{d}:$

$$
\begin{aligned}
|m(x)-\hat{m}(x)| \leq & \left|\sum_{k=1}^{K} g_{k}\left(f_{1, k}(x), \ldots, f_{d^{*}, k}(x)\right)-\sum_{k=1}^{K} g_{k}\left(\hat{f}_{1, k}(x), \ldots, \hat{f}_{d^{*}, k}(x)\right)\right| \\
& +\left|\sum_{k=1}^{K} g_{k}\left(\hat{f}_{1, k}(x), \ldots, \hat{f}_{d^{*}, k}(x)\right)-\sum_{k=1}^{K} \hat{g}_{k}\left(\hat{f}_{1, k}(x), \ldots, \hat{f}_{d^{*}, k}(x)\right)\right| \\
\leq & \sum_{k=1}^{K} L \cdot \sum_{j=1}^{d^{*}}\left|f_{j, k}(x)-\hat{f}_{j, k}(x)\right| \\
& +\sum_{k=1}^{K}\left|g_{k}\left(\hat{f}_{1, k}(x), \ldots, \hat{f}_{d^{*}, k}(x)\right)-\hat{g}_{k}\left(\hat{f}_{1, k}(x), \ldots, \hat{f}_{d^{*}, k}(x)\right)\right| .
\end{aligned}
$$

All the $f_{j, k}$ satisfy a $(p, C)$-smooth generalized hierarchical interaction model of order $d^{*}$ and level $l-1$ and respect the requirements of this theorem. Thus, we can choose the approximations $\hat{f}_{j, k} \in \mathcal{H}^{(l-1)}$ according to the induction hypothesis with $\eta_{n}$ replaced by $\frac{\eta_{n}}{2 \cdot d^{*} \cdot K}$. Then each of the terms $\left|f_{j, k}(x)-\hat{f}_{j, k}(x)\right|$ can be bounded by $c_{32} \cdot a_{n}^{N+q+3} \cdot M_{n}^{-p}$ for all $n$ sufficiently large and $x \in\left[-a_{n}, a_{n}\right]^{d}$ outside of a set $D_{j, k}$ of $\mathbf{P}_{X}$-measure less 
than or equal to $\frac{c}{2 \cdot d^{*} \cdot K} \cdot \eta_{n}$.

Furthermore, let $\hat{f}_{k}: \mathbb{R}^{d} \rightarrow \mathbb{R}^{d^{*}}$ be characterized by $\hat{f}_{k}(x)=\left(\hat{f}_{1, k}(x), \ldots, \hat{f}_{d^{*}, k}(x)\right)^{T}$ and set $\bar{f}_{k, \max }=\max _{j=1, \ldots, d^{*}}\left\|f_{j, k}\right\|_{\infty}$ for all $k=1, \ldots, K$. Given that $c_{32} \cdot a_{n}^{N+q+3} \cdot M_{n}^{-p} \leq c_{32}$ for all sufficiently large $n$ because of the assumptions of Theorem $3, \hat{f}_{k}(x)$ falls into

$$
\hat{F}_{k}=\left[-\bar{f}_{k, \max }-c_{32}, \bar{f}_{k, \max }+c_{32}\right]^{d^{*}}
$$

for all $x \in\left[-a_{n}, a_{n}\right]^{d}$ outside of the union of the sets $D_{j, k}\left(j=1, \ldots, d^{*}, k=1, \ldots, K\right)$ and $n$ sufficiently large. Applying Theorem 2 (if the condition $\bar{f}_{k, \max }+c_{32} \geq 1$ is not satisfied, modify $c_{32}$ adequately) with $\eta=\frac{c \cdot \eta_{n}}{2 \cdot K}$, it is possible to choose a neural network $\hat{g}_{k}$ for every $g_{k}$ in the second sum with a maximum approximation error of

$$
c_{13} \cdot\left(\bar{f}_{k, \max }+c_{32}\right)^{N+q+3} \cdot M_{n}^{-p} \leq c_{33} \cdot M_{n}^{-p}
$$

on $\hat{F}_{k}$ outside of a set $\tilde{D}_{k}$ that satisfies $\mathbf{P}_{\hat{f}_{k}(X)}\left(\tilde{D}_{k}\right) \leq \frac{\eta_{n}}{2 \cdot K}$. For $n$ sufficiently large, the weights of $\hat{g}_{k}$ according to the notation of 2 satisfy

$$
\begin{aligned}
\left|\mu_{i}\right| & \leq c_{14} \cdot\left(\bar{f}_{k, \max }+c_{32}\right)^{q} \cdot M_{n}^{N \cdot p} \leq \alpha, \\
\left|\lambda_{i, l}\right| & \leq M_{n}^{d^{*}+p \cdot(N+2)} \leq \alpha, \\
\left|\theta_{i, l, v}\right| & \leq 6 \cdot d \cdot \frac{1}{\eta_{n}} \cdot M_{n}^{d^{*}+p \cdot(2 N+3)+1} \leq \alpha,
\end{aligned}
$$

which implies $\hat{g}_{k} \in \mathcal{F}_{M^{*}, d^{*}, d^{*}, \alpha}^{(\text {neural notws })}$. Since $\mathbf{P}_{\hat{f}_{k}(X)}\left(\tilde{D}_{k}\right)=\mathbf{P}_{X}\left(\hat{f}_{k}^{-1}\left(\tilde{D}_{k}\right)\right), \hat{g}_{k}\left(\hat{f}_{k}(x)\right)$ approximates $g_{k}\left(\hat{f}_{k}(x)\right)$ with the above maximum error for all

$$
x \in\left[-a_{n}, a_{n}\right]^{d} \backslash \bigcup_{j=1, \ldots, d^{*}} D_{j, k}
$$

outside of a set $D_{k}=\hat{f}_{k}^{-1}\left(\tilde{D}_{k}\right)$ of $\mathbf{P}_{X}$-measure less than or equal to $\frac{c \cdot \eta_{n}}{2 \cdot K}$. Choosing $t(x)=\hat{m}(x)=\sum_{k=1}^{K} \hat{g}_{k}\left(\hat{f}_{1, k}(x), \ldots, \hat{f}_{d^{*}, k}(x)\right)$ as described, we can conclude from $\hat{g}_{k} \in \mathcal{F}_{M^{*}, d^{*}, d^{*}, \alpha}^{(\text {eural networks) }}$ and $\hat{f}_{j, k} \in \mathcal{H}^{(l-1)}$ for all $j=1, \ldots, d^{*}$ and $k=1, \ldots, K$ that $t \in \mathcal{H}^{(l)}$ is valid and that for a sufficiently large $n$

$$
|t(x)-m(x)| \leq K \cdot L \cdot d^{*} \cdot c_{32} \cdot a_{n}^{N+q+3} \cdot M_{n}^{-p}+K \cdot c_{33} \cdot M_{n}^{-p} \leq c_{29} \cdot a_{n}^{N+q+3} \cdot M_{n}^{-p}
$$

holds for all $x \in\left[-a_{n}, a_{n}\right]^{d}$ outside of the union of all exceptional sets so far. The $\mathbf{P}_{X}$-measure of this union satisfies

$$
\mathbf{P}_{X}\left(\bigcup_{\substack{j=1, \ldots, d^{*}, k=1, \ldots, K}} D_{j, k} \cup \bigcup_{k=1, \ldots, K} D_{k}\right) \leq \sum_{\substack{j=1, \ldots, d^{*}, k=1, \ldots, K}} \mathbf{P}_{X}\left(D_{j, k}\right)+\sum_{k=1, \ldots, K} \mathbf{P}_{X}\left(D_{k}\right)
$$




$$
\begin{aligned}
& \leq \sum_{\substack{j=1, \ldots, d^{*}, k=1, \ldots, K}} \frac{c \cdot \eta_{n}}{2 \cdot d^{*} \cdot K}+\sum_{k=1, \ldots, K} \frac{c \cdot \eta_{n}}{2 \cdot K} \\
& =c \cdot \eta_{n},
\end{aligned}
$$

which proves the first assertion of the theorem when $l>0$. The second assertion can be shown analogously to the case of $l=0$ by

$$
\begin{aligned}
|t(x)| & \leq K \cdot\left(\begin{array}{c}
d^{*}+N \\
d^{*}
\end{array}\right) \cdot(N+1) \cdot\left(M_{n}+1\right)^{d^{*}} \cdot \max _{k=1, \ldots, K} c_{14} \cdot\left(\bar{f}_{k, \max }+c_{32}\right)^{q} \cdot M_{n}^{N \cdot p} \\
& \leq c_{34} \cdot M_{n}^{d^{*}+N \cdot p}
\end{aligned}
$$

for all $x \in \mathbb{R}^{d}$, which is an even stronger bound than the announced.

\section{References}

[1] Abramovitz, M., and Stegun, I. A. (1972). Handbook of mathematical functions. Dover Publications, New York, US.

[2] Bauer, B., Heimrich, F., Kohler, M., and Krzyżak, A. (2017). On estimation of surrogate models for high-dimensional computer experiments. To appear in A.. Inst. Statist. Math.

[3] Kohler, M. (2014). Optimal global rates of convergence for noiseless regression estimation problems with adaptively chosen design. Journal of Multivariate Analysis, 132, pp. 197-208.

[4] Mhaskar, H. N. (1993). Approximation properties of a multilayer feedforward artificial neural network. Advances in Computational Mathematics, 1, pp. 61-80.

[5] Sauer, T. (2006). Polynomial interpolation in several variables: lattices, differences, and ideals. In: Jetter, K., Buhmann, M. D., Haussmann, W., Schaback, R., and Stöckler, J. (eds.), Studies in Computational Mathematics, Topics in Multivariate Approximation and Interpolation, 12, pp. 191-230.

[6] Scarselli, F., and Tsoi, A. C. (1998). Universal Approximation Using Feedforward Neural Networks: A Survey of Some Existing Methods, and Some New Results. Neural Networks, 11, pp. 15-37. 